\title{
Relativistic neutron star merger simulations with non-zero temperature equations of state
}

\section{Variation of binary parameters and equation of state ${ }^{\star}$}

\author{
R. Oechslin, H.-T. Janka, and A. Marek
}

\begin{abstract}
Max-Planck-Institut für Astrophysik, Karl-Schwarzschild-Str. 1, 85741 Garching, Germany
e-mail: roe@mpa-garching.mpg.de
\end{abstract}

Received 2 November 2006 / Accepted 21 February 2007

\begin{abstract}
An extended set of binary neutron star (NS) merger simulations is performed with an approximative treatment of general relativity to systematically investigate the influence of the nuclear equation of state (EoS), the NS masses, and the NS spin states prior to merging. The general relativistic hydrodynamics simulations are based on a conformally flat approximation to the Einstein equations and a Smoothed Particle Hydrodynamics code for the gas treatment. We employ the two non-zero temperature EoSs of Shen et al. (1998a, Nucl. Phys. A, 637, 435; 1998b, Prog. Theor. Phys., 100, 1013) and Lattimer \& Swesty (1991, Nucl. Phys. A, 535, 331), which represent a "harder" and a "softer" behavior, respectively, with characteristic differences in the incompressibility at supernuclear densities and in the maximum mass of nonrotating, cold neutron stars. In addition, we use the cold EoS of Akmal et al. (1998, Phys. Rev. C, 58, 1804) with a simple ideal-gas-like extension according to Shibata \& Taniguchi (2006, Phys. Rev. D, 73, 064027), in order to compare with their results, and an ideal-gas EoS with parameters fitted to the supernuclear part of the Shen-EoS. We estimate the mass sitting in a dilute "torus" around the future black hole $(\mathrm{BH})$ by requiring the specific angular momentum of the torus matter to be larger than the angular momentum of the ISCO around a Kerr BH with the mass and spin parameter of the compact central remnant. The dynamics and outcome of the models is found to depend strongly on the EoS and on the binary parameters. Larger torus masses are found for asymmetric systems (up to $\sim 0.3 M_{\odot}$ for a mass ratio of 0.55 ), for large initial NSs, and for a NS spin state which corresponds to a larger total angular momentum. We find that the postmerger remnant collapses either immediately or after a short time when employing the soft EoS of Lattimer\& Swesty, whereas no sign of post-merging collapse is found within tens of dynamical timescales for all other EoSs used. The typical temperatures in the torus are found to be about 3-10 MeV depending on the strength of the shear motion at the collision interface between the NSs and thus depending on the initial NS spins. About $10^{-3}-10^{-2} M_{\odot}$ of NS matter become gravitationally unbound during or right after the merging process. This matter consists of a hot/high-entropy component from the collision interface and (only in case of asymmetric systems) of a cool/low-entropy component from the spiral arm tips.
\end{abstract}

Key words. stars: neutron - gamma rays: bursts - hydrodynamics - equation of state - methods: numerical

\section{Introduction}

Merger events of double neutron star (DNS) binaries do not only belong to the strongest known sources of gravitational wave (GW) radiation, they are also widely favored as origin for the class of short, hard GRBs (Blinnikov et al. 1984; Paczyński 1986; Eichler et al. 1989; Paczyński 1991; Narayan et al. 1992; Oechslin \& Janka 2006). This possible link seems to be supported by recent short GRBs with determined redshift (see e.g. Gehrels et al. 2005; Fox et al. 2005; Berger et al. 2005). Theoretical gravitational waveforms from simulations are needed in the future detection process as templates for filtering out the actual signal from the noisy detector output. The gravitational wave signal of the late inspiral and merging phases contains information on the nuclear EoS and on the binary parameters such as NS masses and spins.

Knowledge about the merging is also needed as a basic ingredient for theoretical short GRB models (Aloy et al. 2005).

* Appendices are only available in electronic form at http://www . aanda. org
The post-merging black hole (BH)-torus configuration serves as initial data for such models and thus, information about the torus parameters is essential in this context.

Prior to coalescence, the DNS binary spirals in due to energy and angular momentum loss by gravitational radiation. This inspiral phase lasts millions of years and can be very accurately modelled in the post-Newtonian framework (e.g. Damour et al. 2001, 2002). In contrast, the coalescence itself happens on a dynamical timescale of milliseconds and simulating such a process naturally involves three-dimensional general relativistic hydrodynamics. In addition, different aspects of physics enter the problem at some stage. Due to the high compactness, gravitational interaction has to be described by full general relativity (GR). Nuclear and particle physics enter in the description of the hot and dense neutron star fluid via an equation of state (EoS) and in the treatment of the neutrino generation after the actual merging event. Finally, the possible formation of heavy elements is of interest when matter gets ejected during the coalescence.

Up to now, attempts to investigate the process have concentrated either on the relativistic aspects while greatly simplifying 
the microphysics, or a nuclear physics based EoS was used together with an approximative neutrino transport while describing gravity in a Newtonian framework. The field was pioneered by the work of Oohara \& Nakamura (1989). Relativistic aspects have been considered in Oohara \& Nakamura (1992); Ayal et al. (2001); Faber \& Rasio (2000) and Faber \& Rasio (2002) in the post-Newtonian (PN) framework, which however breaks down when gravity becomes too strong. The conformal flatness approach, a middle ground between PN and full general relativity (GR), has been chosen in Wilson et al. (1996); Oechslin et al. (2002, 2004); Faber et al. (2004), and Oechslin \& Janka (2006). A fully relativistic treatment has been adopted by Shibata \& Uryū (2002); Shibata et al. (2005), and Shibata \& Taniguchi (2006).

On the other hand, Newtonian simulations with microphysical improvements have been achieved in Ruffert et al. (1996); Rosswog et al. (1999); Ruffert \& Janka (2001), and Rosswog $\&$ Liebendörfer (2003). These works implemented either the nuclear-physics based, non-zero-temperature equation of state (EoS) of Lattimer \& Swesty (1991) or the one of Shen et al. (1998a,b). Furthermore, neutrino physics has been implemented using a neutrino-trapping scheme.

First steps to unite these two "branches" have been taken on the one hand by Oechslin et al. (2004), who considered within the conformal flatness approximation to GR the influence of different microphysical zero-temperature EoSs on the inspiral and merger dynamics, and, on the other hand by Shibata et al. (2005) and Shibata \& Taniguchi (2006), who implemented besides full GR an analytic fit to several physical zero-temperature EoSs together with an ideal-gas-like extension that approximatively accounts for finite-temperature effects.

Using an extended set of hydrodynamics simulations, the present work studies the dependence of the merging and the subsequent postmerger evolution on the nuclear EoS and on the binary parameters like the NS masses and the initial NS spins. Similar aims, in Newtonian and post-Newtonian gravity, but without such combination of GR and detailed microphysics have been pursued by several authors (Rosswog et al. 2000; Faber et al. 2001; Faber \& Rasio 2002), mainly focusing on the gravitational wave signal and the amount of gravitationally unbound matter.

Our paper is organized as follows. In Sect. 2 and in Appendix A, we sketch the formalism on which our simulations are based and its implementation in our numerical scheme. In Sect. 3, the different initial models for our simulations and their construction is described. The results are presented in Sect. 4. Finally, our conclusions are summarized in Sect. 5.

\section{The formalism}

\subsection{Hydrodynamics}

We employ an improved version of our relativistic smoothed particle hydrodynamics (SPH) code (Oechslin et al. 2002, 2004), which solves the relativistic hydrodynamics equations together with the Einstein field equations in the conformally flat approximation (CFC; Isenberg \& Nester 1980; Wilson et al. 1996). The detailed formalism and its implementation are sketched in Appendix A.

The improved code version now includes two finitetemperature nuclear EoSs, namely the ones of Shen et al. (1998a,b) and of Lattimer \& Swesty (1991). They allow for a microphysically-based treatment of matter from low densities to well above nuclear saturation density. Their non-zero-temperature character also allows for an investigation of the thermal evolution in regions where shock heating is present. We have also implemented the new SPH artificial viscosity (AV) formalism of Chow \& Monaghan (1997). It replaces the "standard" formalism (Monaghan 1992) and the one proposed by Siegler \& Riffert (2000), which were used in our old code version. The AV interaction is determined in Chow \& Monaghan (1997) by approximately solving a Riemann problem between particle neighbours.

The revised backreaction formalism mimicking the energy and angular momentum dissipation by gravitational waves now follows the lines of the formalism of Blanchet et al. (1990, BDS), but includes some higher-order terms as given by Faye $\&$ Schäfer (2003) (see Appendix A). The reactive contribution to the metric coefficients is added to the $\mathrm{CFC}$-approximated metric terms.

Neutrino physics is not implemented as we consider the backreaction of neutrino emission on the fluid as negligeable on timescales on the order of $\sim 10 \mathrm{~ms}$ considered here. As a consequence, the electron fraction $Y_{\mathrm{e}}$, once determined from the initial conditions, does not change within a fluid element and is simply advected with the moving fluid.

\subsection{The equations of state}

We consider a set of four different EoSs:

- the EoS of Shen et al. (1998a,b, "Shen-EoS");

- the EoS of Lattimer \& Swesty (1991, "LS-EoS");

- the EoS of Akmal et al. (1998, "APR-EoS") extended with an ideal gas-like thermal contribution; and

- an ideal-gas EoS with adiabatic index and adiabatic constant fitted to the supernuclear part of the Shen-EoS.

The Shen-EoS uses a phenomenological relativistic mean field (RMF) approach which aims at reproducing results from Relativistic Brückner-Hartree-Fock (RBHF) calculations. It has a relatively high incompressibility modulus of $K_{0}=280 \mathrm{MeV}$ and thus leads to large NS-radii and maximum masses (see Fig. 2). We implement the EoS in tabulated form with $\rho$ (baryonic density), $T$ (temperature), and $Y_{\mathrm{e}}$ (electron fraction) as input quantities. Since our hydro code evolves the specific internal energy $\epsilon$ rather than the temperature (via the energy Eq. (A.15) and the relation (A.20) in Appendix A), the EoS has to be evaluated implicitly for given $\rho, Y_{\mathrm{e}}$, and $\epsilon$.

The LS-EoS employs a finite-temperature compressible liquid droplet model for nuclei (Lattimer et al. 1985). Our version of the LS-EoS has been constructed using an incompressibility modulus of $K_{0}=180 \mathrm{MeV}$, thus generally providing lower pressure values than the Shen-EoS for given $\rho, T$, and $Y_{\mathrm{e}}$. Consequently, the NS radii are much smaller and the maximum NS mass allowed by the LS-EoS is marginally compatible with the NS mass of $2.1 \pm 0.2 M_{\odot}$ recently measured by Nice et al. (2005). The numerical implementation of the LS-EoS in our code is similar to the one of the Shen-EoS.

The APR-EoS is implemented as in Shibata \& Taniguchi (2006) in order to allow for a comparison of our results with theirs. The pressure and specific internal energy are both written as a sum of a thermal and a cold contribution. The cold component of the specific internal energy $\epsilon_{\text {cold }}$ is a function of $\rho$ and is given by an analytic fit formula to the table values provided by Akmal et al. (1998), who used the variational chain summation method together with the Argonne $v_{18}$ nucleonnucleon interaction model. Three-nucleon-interactions and relativistic boost corrections are also considered. The cold pressure 


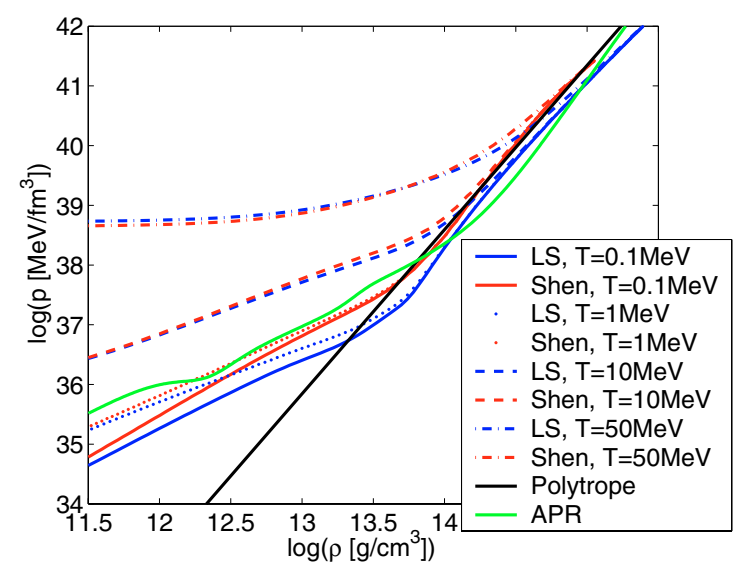

Fig. 1. Pressure versus density for a set of representative temperatures for the two considered physical EoSs of Lattimer \& Swesty (1991) and of Shen et al. (1998a,b). The electron fraction is set to $Y_{\mathrm{e}}=0.05$. For comparison, we also show the polytropic relation $p=K \rho^{\Gamma}$ used in this work with $K=30000, \Gamma=2.75$ and the APR-EoS for $T=0$.

component is not directly read from the EoS table but is determined by the thermodynamic relation $p_{\text {cold }}=\rho^{2} \mathrm{~d} \epsilon_{\text {cold }} / \mathrm{d} \rho$. The thermal pressure component $p_{\text {th }}$ is given by the ideal-gas law $p_{\mathrm{th}}=\left(\Gamma_{\mathrm{th}}-1\right) \rho \epsilon_{\mathrm{th}}$, where the specific thermal energy is given by $\epsilon_{\mathrm{th}}=\epsilon-\epsilon_{\mathrm{cold}}$ and the adiabatic index $\Gamma_{\mathrm{th}}$ determines the dependence of the pressure on the internal energy at constant density and is chosen to be $\Gamma_{\text {th }}=1.5$ or $\Gamma_{\text {th }}=2$. Thus, the total pressure $p$ is determined from the input quantities $\rho$ and $\epsilon$.

The APR-EoS has the specific property of being rather soft around nuclear matter density while being very stiff at much higher densities (Fig. 1). This leads to very compact NSs (Fig. 3) but still allows for a fairly large maximum NS mass (Fig. 2). This behaviour is significantly different from that obtained with the other EoSs. It will also lead to some peculiarities of the APR models of NS mergers.

The ideal-gas EoS is given by the simple relation $p=$ $(\Gamma-1) \rho \epsilon$, where a polytropic relation $\epsilon=K /(\Gamma-1) \rho^{\Gamma-1}$ is used to initialize the internal energy. The parameters $\Gamma=2.75$ and $K=30000$ (in geometrical units) are chosen such that the relation between central density and NS mass of the Shen-EoS is reproduced for a wide range of NS masses (see Fig. 2). This leads to an EoS that not only closely fits the supernuclear regime of the Shen-EoS at $T \sim 0$ (see Fig. 1) but also yields very similar NS density profiles (see Fig. 3). However, since these EoSs deviate from each other at subnuclear densities when nuclear forces become unimportant (see Fig. 1), we can investigate the importance of this regime of the EoS during NS coalescence.

We also explore the importance of the non-zero temperature effects either by varying the parameter $\Gamma_{\text {th }}$ in the APR-EoS or by considering cases with the Shen-EoS, where we artificially neglect the thermal component in the internal energy. This is done by restricting the full EoS to the two-dimensional subtable at $T=0$ with density and electron fraction as remaining independent variables and pressure and internal energy as output quantities ("cold Shen-EoS"). The energy Eq. (A.15) is not needed in this case. This reduction has no influence as long as shocks and viscous heating are absent, i.e. as long as the fluid evolves adiabatically. In the presence of shock heating or viscous heating, however, it corresponds to the extreme case that a very efficient cooling mechanism extracts immediately the generated entropy and internal energy.

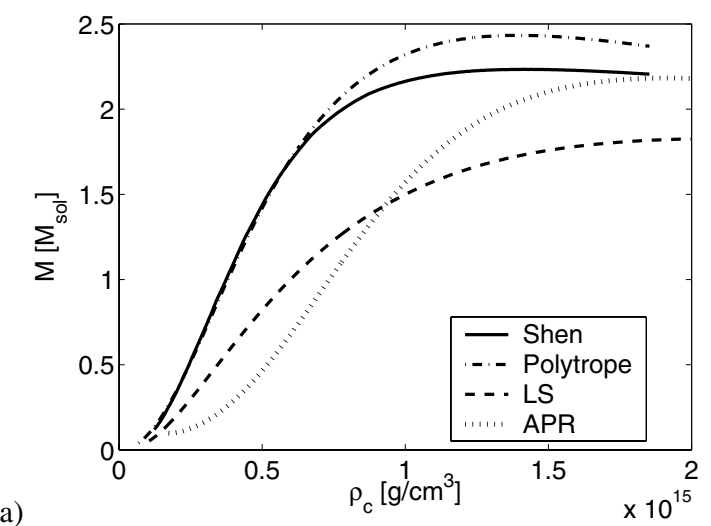

(a)

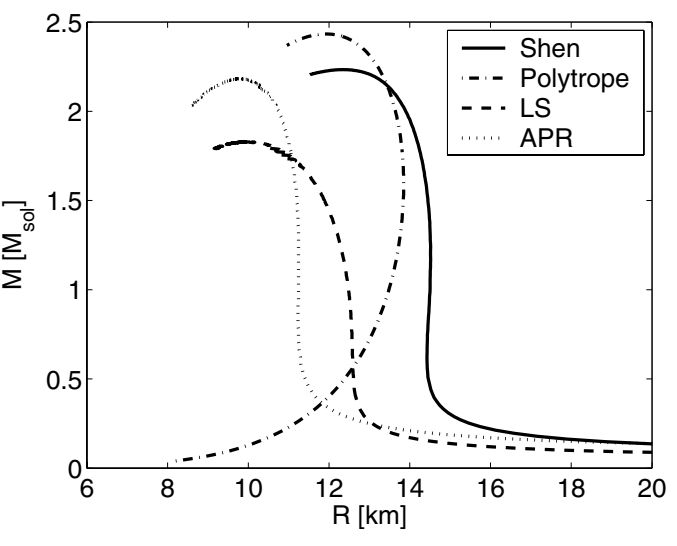

Fig. 2. ADM NS mass $M$ versus central density $\rho_{\mathrm{c}}$ (panel a)) and versus circumferential radius $R$ (panel b)) for the Shen-EoS (solid) and the LS-EoS (dashed) with $T=0.1 \mathrm{MeV}$, for the APR-EoS (dotted) and for the polytropic EoS (dash-dotted). The parameters $K$ and $\Gamma$ of the latter are chosen such that the $\rho_{\mathrm{c}}-M$ - relation of the Shen-EoS is reproduced in a wide range of NS masses up to $M \sim 1.8 M_{\odot}$. The difference between the two EoSs in the $R-M$ relation is due to differences in the low-density regime, which are crucial for determining the NS radius.

\subsection{Estimation of the postmerger torus mass}

Provided the central, compact merger remnant has a mass above a certain critical value, it will collapse to a $\mathrm{BH}$, either immediately after merging or with some delay, after sufficient angular momentum has been carried away by gravitational radiation or has been redistributed to matter at larger radii by processes like non-axisymmetric gravitational interactions, viscosity, and magnetic field effects (Morrison et al. 2004). Therefore, any pressure support from the central remnant to the surrounding halo gas will vanish and matter will be kept from infall to the $\mathrm{BH}$ mostly by stabilizing rotational support.

Consequently, a lower estimate for the mass remaining in a halo around the $\mathrm{BH}$ is given by the condition

$\hat{u}_{\phi}>j_{\mathrm{ISCO}}$

i.e., we demand the specific angular momentum $\hat{u}_{\phi}$ of a fluid element in the halo (called "torus" in the following) to be larger than the angular momentum at the innermost stable circular orbit (ISCO) of a Kerr BH with the same mass and angular momentum as the compact merger remnant.

For a Boyer-Lindquist Kerr metric (Bardeen et al. 1972), the ISCO can be analytically determined. In our case, however, we shall use an approximative pseudo-Kerr metric (Grandclément et al. 2002) which is both isotropic and conformally flat, consistent with the coordinates we use for the numerical simulations. 
The ISCO can then be found among all circular orbits by minimizing the specific angular momentum along the radial coordinate. The detailed calculation is presented in Appendix $\mathrm{C}$.

\section{Initial conditions}

\subsection{Construction of the initial data}

We start our simulations shortly before the tidal instability occurs and follow the evolution through the merging and torus formation until either the gravitational collapse of the merger remnant to a $\mathrm{BH}$ sets in or a quasi-stationary state has formed.

At the beginning of the simulations, we have to provide initial values for the primitive variables $\rho, \epsilon$, and $Y_{\mathrm{e}}$ (in the cases of the Shen-EoS and LS-EoS).

In a first step, we couple the latter two variables uniquely to the density using the following conditions: In the Shen-EoS and LS-EoS case, we assume the two NSs to be cold and in neutrinoless beta-equilibrium, i.e. for the neutrino chemical potential the relation $\mu_{v}=\mu_{\mathrm{n}}-\mu_{\mathrm{p}}-\mu_{\mathrm{e}}=0$ holds. Together with the condition $T \sim 0$, this fixes uniquely the electron fraction $Y_{\mathrm{e}}$ and the specific internal energy $\epsilon$ for a given density $\rho^{1}$. In the APR-EoS, we implement the " $T \sim 0$ "-condition by requiring $\epsilon=\epsilon_{\text {cold }}$. In the case of the ideal-gas EoS, we use the polytropic relation $\epsilon=K /(\Gamma-1) \rho^{\Gamma-1}$ in order to provide the initial internal energy. Together with the ideal-gas relation, we then obtain the familiar expression $p=K \rho^{\Gamma}$ for the initial pressure. As described in Sect. 2.2, we choose the parameters $K$ and $\Gamma$ such that the central density-mass relation of the Shen-EoS for a wide range of NS masses (see Fig. 2) is reproduced. In all EoS cases we then obtain a unique relation between density, pressure, and specific internal energy ("1D-EoS").

In a second step, we use the Oppenheimer-Volkoff-Equation in isotropic coordinates together with the above described 1D EoSs to obtain radial NS profiles of the hydrodynamic quantities and the space time metric. We then generate SPH particle distributions of the two merging NSs in isolation by placing particles on cylindrical shells around the $z$-axis to maintain axisymmetry. The mutual distance between particles is chosen in order to approximatively obtain a prescribed number of particles. The particle masses and smoothing lengths are adjusted such that the particle densities approximate the earlier obtained OV density profile and such the number of SPH neighbour particles remains within prescribed limits, typically between 80 and 120 neighbours.

Finally, the two NSs are placed on a circular orbit with a chosen orbital distance such that the common center of rest mass $\boldsymbol{r}_{\mathrm{CM}}=\int \rho^{*} \boldsymbol{r} \mathrm{d}^{3} x / \int \rho^{*} \mathrm{~d}^{3} x$ is located in the coordinate origin. Here, $\rho^{*}$ is the conserved density as defined in Eq. (A.18) and $\boldsymbol{r}$ is the position vector. The orbital separation is defined as the coordinate distance between the stellar centers of rest mass, $\boldsymbol{r}_{\mathrm{CM}, i}=\int_{\operatorname{star} i} \rho^{*} \boldsymbol{r} \mathrm{d}^{3} x / \int_{\operatorname{star} i} \rho^{*} \mathrm{~d}^{3} x$. This system is then relaxed with the evolution code to an equilibrium configuration by adding a braking term $f_{\text {brake }}$ to the momentum equation,

$\boldsymbol{f}_{\text {brake }}=C\left(\hat{\boldsymbol{u}}-\hat{\boldsymbol{u}}_{\mathrm{eq}}\right)$,

where $C$ is a chosen small constant, $\hat{\boldsymbol{u}}$ the current specific momentum (see Appendix A for definitions) and $\hat{\boldsymbol{u}}_{\text {eq }}$ the specific

\footnotetext{
${ }^{1}$ Note that in the case of our tabulated version of the LS-EoS, the beta-equilibrium condition has no solution in a small region around nuclear saturation density and at very low temperatures. In this case, we look for an approximate solution by minimizing $\mu_{v}$ along the $Y_{\mathrm{e}}$ variable, obtaining $Y_{\mathrm{e}} \simeq 0.02$.
}

momentum field to be relaxed to. We set

$\hat{\boldsymbol{u}}_{\mathrm{eq}}=\hat{\boldsymbol{u}}_{\mathrm{orbit}}+\hat{\boldsymbol{u}}_{\mathrm{spin}}$,

where

$\hat{\boldsymbol{u}}_{\mathrm{orbit}, i}=\hat{\mathbf{\Omega}}_{\mathrm{eq}} \times \boldsymbol{r}_{\mathrm{CM}, i}$

describes the equilibrium orbital motion and

$\hat{\boldsymbol{u}}_{\mathrm{spin}, i}=\hat{\mathbf{\Omega}}_{i} \times\left(\boldsymbol{r}-\boldsymbol{r}_{\mathrm{CM}, i}\right)$

is the contribution from the stellar rotation. Here, the quantity $\hat{\mathbf{\Omega}}$ denotes to the angular velocity related to $\hat{\boldsymbol{u}}$ (defined in analogy to the angular velocity $\Omega$ related to the physical velocity $\boldsymbol{v}$ ). More specifically, $\hat{\boldsymbol{\Omega}}_{i}$ and $\hat{\boldsymbol{\Omega}}_{\mathrm{eq}}$ refer to the angular velocity related to $\hat{\boldsymbol{u}}$ of the individual NSs and of the orbital motion of the binary, respectively. For an irrotating spin setup, we set $\hat{\mathbf{\Omega}}_{i}=0$, for a corotating one $\hat{\mathbf{\Omega}}_{i}=\hat{\mathbf{\Omega}}_{\mathrm{eq}}$, and for a counterrotating spin setup $\hat{\mathbf{\Omega}}_{i}=-\hat{\mathbf{\Omega}}_{\mathrm{eq}}$. Finally, the setup with opposite NS rotation means $\hat{\mathbf{\Omega}}_{1}=\hat{\mathbf{\Omega}}_{\mathrm{eq}}=-\hat{\mathbf{\Omega}}_{2}$. Now, $\hat{\mathbf{\Omega}}_{\mathrm{eq}}$ (and thus the $\hat{\mathbf{\Omega}}_{i}$ ) has to be determined such that the mutual gravitational attraction and the centrifugal forces are in orbital equilibrium. Starting from a guess value, we do this iteratively during the relaxation process by increasing $\hat{\boldsymbol{\Omega}}_{\mathrm{eq}}$ if the NSs tend to fall towards each other while decreasing it if the orbital separation tends to grow. At the same time, the orbital distance is reset to the initially chosen value.

Using $C=-0.02$, global quantities like the total angular momentum $J$ and the orbital angular velocity $\Omega$ (both defined in Appendix B) settle down to stable values within roughly two orbital periods. Subsequent oscillations at the $1 \%$ level (see Fig. 4) are mainly due to readjustments of the orbital distance during the relaxation process. We usually stop the calculation after about four orbital periods and read off the initial data at the end.

The definition of an irrotational velocity field by $\hat{\boldsymbol{\Omega}}_{i}=0$ is motivated from Newtonian physics. It satifies the equations used by the helical killing vector (HKV) method (Shibata 1998; Gourgoulhon et al. 2001) to Newtonian order. To investigate the error we introduce with our approximative definition of irrotationality, we compare the obtained results to quasi-equilibrium models based on the HKV method (Uryū \& Eriguchi 2000; Gourgoulhon et al. 2001). We typically find devitations in the angular momentum $J$ and the angular velocity $\Omega$ of the order of $1 \%$ (Fig. 4). Note that we could also have used the physical velocity $\mathbf{v}$ rather than the specific momentum $\hat{\boldsymbol{u}}$ for the definition of the equilibrium velocity field since the latter two quantities are equal to Newtonian order. However, the use of the specific momentum yields a much better agreement in $J$ and $\Omega$ with results obtained with the HKV method.

\subsection{Initial models}

In order to study the influence of the various parameters that characterize the initial model and to determine the subsequent merger dynamics, we vary the individual NS masses $M_{1}$ and $M_{2}$, the NS spin setup, and the EoS. In Table 1 we summarize the describing parameters of our models.

We consider both equal-mass models, i.e. with a mass ratio $q=M_{1} / M_{2}=1$, and unequal-mass models in order to investigate the consequences of $q<1$. A mass ratio near unity seems to be favoured by observations, but population synthesis models suggest that beside a population component of systems with nearly equal masses also a significant fraction of binaries with $q$ as low as 0.55 might exist (Bulik et al. 2003). 
Table 1. Characteristic parameters and data of our considered models. $M_{1}$ and $M_{2}$ denote the individual gravitational masses in isolation while $M_{\text {sum }}=M_{1}+M_{2}$ stands for the sum of the two (Note that the total gravitational mass $M$ is slightly smaller than $M_{\text {sum }}$ because $M$ also involves the negative gravitational binding energy.). $M_{0}$ is the total baryonic mass, whereas $q=M_{1} / M_{2}$ and $q_{M}=M_{0,1} / M_{0,2}$ are the gravitational and baryonic mass ratio, respectively. "Shen" stands for the full, finite temperature Shen-EoS, "Shen_c" for the restriction to zero temperatures, "LS" denotes the LS-EoS, "ideal" means ideal-gas EoS, and, finally, "APR15" and "APR2" refer to the APR-EoS extended using $\Gamma_{\text {th }}=1.5$ and $\Gamma_{\text {th }}=2$, respectively. The NS spin states are denoted as "irrot" for irrotating, "corot" for corotating, "c_rot" for counterrotating, "oppo" for oppositely oriented spins and "tilted" for a spin orientation inclined relative to the orbital spin. More specifically, we choose in model S1414t1 the angular velocity vectors $\hat{\mathbf{\Omega}}_{1}=0$ and $\hat{\mathbf{\Omega}}_{2}=0.041 *(0,1,0)$ (in geometrical units), which corresponds to a spin period of the second NS of about $1 \mathrm{~ms}$, while in model S1414t2, we set $\hat{\mathbf{\Omega}}_{1}=0.01 *(0,1,1)$ and $\hat{\mathbf{\Omega}}_{2}=0.01 *(0,-1,1) . T_{\max }$ is the maximum temperature reached in the system during the whole evolution. It is obtained by averaging the particle temperatures on a grid with cells of $1.5 \mathrm{~km}$ side length. The rotation parameters $a_{\mathrm{ini}}=J_{\mathrm{ini}} / M_{\mathrm{ini}}^{2}$, $a_{\text {system }}$, and $a_{\text {remnant }}$ refer to the initial binary model, the entire system right after merging, and to only the compact merger remnant at that time. Finally, $M_{\text {torus }}$ and $M_{\mathrm{ej}}$ denote the (baryonic) torus mass and ejecta mass about $5 \mathrm{~ms}$ after merging when a quasi-stationary state has formed.

\begin{tabular}{|c|c|c|c|c|c|c|c|c|c|c|c|c|c|c|c|}
\hline 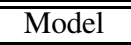 & 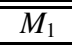 & 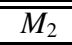 & $\overline{M_{\text {sum }}}$ & $\overline{M_{0}}$ & $\overline{q q}$ & $\overline{q q_{M}}$ & EoS & \multicolumn{2}{|c|}{$\overline{\text { Spins }}$} & $\overline{T_{\max }}$ & $a_{\text {ini }}$ & $a_{\text {system }}$ & $\overline{a_{\text {remnant }}}$ & $\overline{M_{\text {torus }}}$ & $\overline{M_{\mathrm{ej}}}$ \\
\hline Units & $M_{\odot}$ & $M_{\odot}$ & $M_{\odot}$ & $M_{\odot}$ & & & & & & $\mathrm{MeV}$ & & & & $M_{\odot}$ & $10^{-3} M_{\odot}$ \\
\hline S1414 & 1.4 & 1.4 & 2.8 & 3.032 & 1.0 & 1.0 & Shen & irrot & $x \times$ & 52 & 0.96 & 0.91 & 0.88 & 0.04 & 1.5 \\
\hline $\mathrm{S} 138142$ & 1.38 & 1.42 & 2.8 & 3.032 & 0.97 & 0.97 & Shen & irrot & $x \times$ & 50 & 0.96 & 0.90 & 0.87 & 0.04 & 3 \\
\hline S135145 & 1.35 & 1.45 & 2.8 & 3.034 & 0.93 & 0.93 & Shen & irrot & $x x$ & 50 & 0.96 & 0.90 & 0.86 & 0.07 & 5 \\
\hline S1315 & 1.3 & 1.5 & 2.8 & 3.037 & 0.87 & 0.86 & Shen & irrot & $x x$ & 50 & 0.96 & 0.90 & 0.84 & 0.14 & 6 \\
\hline S1216 & 1.2 & 1.6 & 2.8 & 3.039 & 0.75 & 0.73 & Shen & irrot & $x x$ & 54 & 0.95 & 0.89 & 0.76 & 0.21 & 12 \\
\hline S1515 & 1.5 & 1.5 & 3.0 & 3.274 & 1.0 & 1.0 & Shen & irrot & $x x$ & 67 & 0.94 & 0.89 & 0.86 & 0.04 & -3 \\
\hline S1416 & 1.4 & 1.6 & 3.0 & 3.274 & 0.88 & 0.86 & Shen & irrot & $x x$ & 55 & 0.94 & 0.89 & 0.84 & 0.14 & 7 \\
\hline S1317 & 1.3 & 1.7 & 3.0 & 3.279 & 0.76 & 0.75 & Shen & irrot & $x x$ & 65 & 0.94 & 0.88 & 0.78 & 0.20 & 8 \\
\hline S119181 & 1.19 & 1.81 & 3.0 & 3.289 & 0.66 & 0.63 & Shen & irrot & $x \times$ & 57 & 0.91 & 0.87 & 0.72 & 0.24 & 25 \\
\hline S107193 & 1.07 & 1.93 & 3.0 & 3.306 & 0.55 & 0.52 & Shen & irrot & $x \times$ & 70 & 0.87 & 0.84 & 0.69 & 0.26 & 45 \\
\hline $\mathrm{S} 1313$ & 1.3 & 1.3 & 2.6 & 2.800 & 1.0 & 1.0 & Shen & irrot & $x x$ & 52 & 0.99 & 0.93 & 0.89 & 0.06 & 4 \\
\hline S1214 & 1.2 & 1.4 & 2.6 & 2.799 & 0.86 & 0.85 & Shen & irrot & $x \times$ & 43 & 0.98 & 0.93 & 0.82 & 0.19 & -3 \\
\hline S1115 & 1.1 & 1.5 & 2.6 & 2.807 & 0.73 & 0.71 & Shen & irrot & $x \times$ & 50 & 0.99 & 0.92 & 0.76 & 0.22 & 13 \\
\hline C1216 & 1.2 & 1.6 & 2.8 & 3.039 & 0.75 & 0.73 & Shen_c & irrot & $x \times$ & -1 & 0.95 & 0.90 & 0.75 & 0.28 & 5 \\
\hline C1315 & 1.3 & 1.5 & 2.8 & 3.037 & 0.87 & 0.86 & Shen_c & irrot & $x \times$ & -1 & 0.96 & 0.91 & 0.81 & 0.20 & 3 \\
\hline $\mathrm{P} 1315$ & 1.3 & 1.5 & 2.8 & 3.064 & 0.87 & 0.86 & ideal & irrot & $x \times$ & -1 & 0.96 & 0.90 & 0.84 & 0.14 & 8 \\
\hline LS1414 & 1.4 & 1.4 & 2.8 & 3.077 & 1.0 & 1.0 & LS & irrot & $x x$ & 175 & 0.96 & 0.86 & $0.85^{3}$ & $0.02^{3}$ & 2 \\
\hline LS1216 & 1.2 & 1.6 & 2.8 & 3.087 & 0.75 & 0.73 & LS & irrot & $x \times$ & -2 & 0.95 & $0.85^{2}$ & $0.78^{2}$ & $0.19^{2}$ & -2 \\
\hline A151414 & 1.4 & 1.4 & 2.8 & 3.032 & 1.0 & 1.0 & APR15 & irrot & $x \times$ & -1 & 0.97 & 0.84 & $0.81^{3}$ & $0.06^{3}$ & 2 \\
\hline A21414 & 1.4 & 1.4 & 2.8 & 3.032 & 1.0 & 1.0 & APR2 & irrot & $x \times$ & -1 & 0.97 & 0.84 & $0.83^{3}$ & $0.04^{3}$ & 2 \\
\hline S1414co & 1.4 & 1.4 & 2.8 & 3.032 & 1.0 & 1.0 & Shen & corot & $\uparrow \uparrow$ & 40 & 1.05 & 0.98 & 0.86 & 0.21 & 1 \\
\hline S1414ct & 1.4 & 1.4 & 2.8 & 3.032 & 1.0 & 1.0 & Shen & c_rot & $\downarrow \downarrow$ & 74 & 0.88 & 0.85 & 0.83 & 0.02 & 2.5 \\
\hline S1414o & 1.4 & 1.4 & 2.8 & 3.032 & 1.0 & 1.0 & Shen & oppo & $\uparrow \downarrow$ & 46 & 0.97 & 0.92 & 0.90 & 0.07 & 2 \\
\hline $\mathrm{S} 1414 \mathrm{t} 1$ & 1.4 & 1.4 & 2.8 & 3.032 & 1.0 & 1.0 & Shen & tilted & $\leftarrow \times$ & 59 & 0.99 & 0.95 & 0.85 & 0.20 & 28 \\
\hline $\mathrm{S} 1414 \mathrm{t} 2$ & 1.4 & 1.4 & 2.8 & 3.032 & 1.0 & 1.0 & Shen & tilted & $\nearrow \nwarrow$ & 39 & 1.05 & 0.98 & 0.86 & 0.23 & -3 \\
\hline $\mathrm{S} 1216 \mathrm{co}$ & 1.2 & 1.6 & 2.8 & 3.039 & 0.75 & 0.73 & Shen & corot & $\uparrow \uparrow$ & 48 & 1.03 & 0.98 & 0.78 & 0.28 & 33 \\
\hline S1216ct & 1.2 & 1.6 & 2.8 & 3.039 & 0.75 & 0.73 & Shen & c_rot & $\downarrow \downarrow$ & 73 & 0.87 & 0.84 & 0.74 & 0.18 & 14 \\
\hline
\end{tabular}

${ }^{1}$ EoS does not contain temperature information.

${ }^{2}$ Quantity still changes when the collapse to a BH sets in immediately after merging.

${ }^{3}$ Quantity still increases at the end of the run.

Many of our models are chosen with zero NS spin ("irrotating"), which is generally considered to be a good approximation to reality because the viscous timescale is too large to allow for tidal locking during inspiral and because the orbital period near the ISCO of $2 \mathrm{~ms}$ is considerably smaller than typical NS spin periods (Kochanek 1992; Bildsten \& Cutler 1992). Observed NS spin periods in double NS binaries are indeed roughly ten times larger (Stairs 2004). In view of the large influence of the NS spins on the merger, we still think that a set of representative models with non-zero NS spins is worth being considered.

\section{Results}

In the further analysis and discussion of our models, with have normalized the time axis such that $t=0$ coincides with the time when the gravitational-wave luminosity during merging becomes maximal. This allows for a better comparison of the merger and postmerger evolution of different models.

\subsection{Merger dynamics}

We start our simulations somewhat outside the ISCO, i.e. at an orbital separation where the binary is still stable. In our equilibrium initial models we neglect the radial component of the orbital velocity. Miller (2004) suggests that this leads to a systematic error in the inspiral dynamics and the time until merging. We have performed test calculations with different initial orbital distances and with artificially reduced initial angular momentum and backreaction strength. They indeed show a different inspiral behaviour, but the merger and postmerger phases depend very weakly on the inspiral dynamics. Shibata \& Uryū (2002) arrived at a similar conclusion. Since we concentrate in this work on the merger and postmerger dynamics and on the final outcome, 
(a)
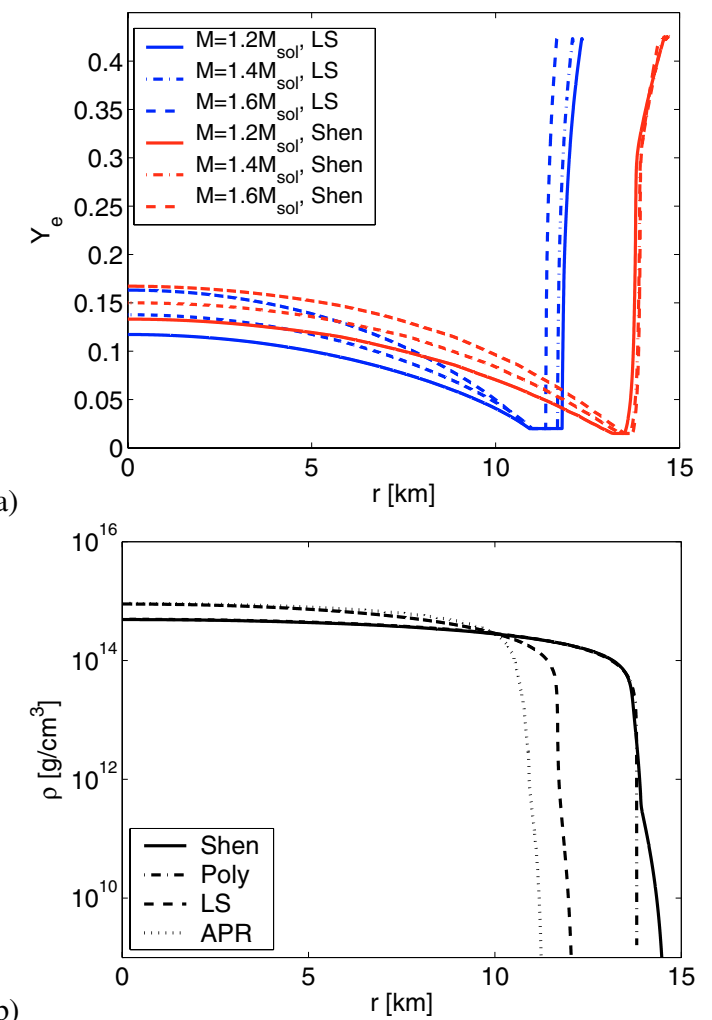

Fig. 3. a) $Y_{\mathrm{e}}$-profiles in NSs with ADM masses of 1.2, 1.4 and $1.6 M_{\odot}$, using both the Shen-EoS and the LS-EoS. The temperature is set to $T=$ $0.1 \mathrm{MeV}$ and neutrinoless beta-equilibrium is assumed (see text). Note that a lower limit of $Y_{\mathrm{e}}=0.02$ had to be set in a small region around nuclear saturation density. b) Density profiles for a representative NS with an ADM mass of $1.4 M_{\odot}$ as computed for the four considered EoSs.

we can safely rely on this approximation. In the following subsections, we are going to investigate the impact of the three parameters - the NS mass ratio, the nuclear EoS, and the initial NS spins - which largely determine the merger dynamics, the remnant properties, and the possible formation of a torus.

\subsubsection{Different mass ratios}

In models with a mass ratio considerably different from unity, e.g. in models S107193, S1216 or S1317, the less massive star is tidally stretched and disrupted, forming a long spiral arm which is accreted to a large part on its more massive companion. This leads to a non-axisymmetric, differentially rotating central object (called "remnant" in the following). The core of the central remnant forms from the more massive NS while the accreted material from the less massive partner is wound up to end in the outer shells of the remnant. Due to a "sling-shot-effect" in the spiral arm, a significant amount of angular momentum is carried from the remnant to larger radii such that the material sitting in the spiral arm tip manages to escape accretion and to contribute to the main part of a future torus (for a detailed discussion, see Oechslin \& Janka 2006). A small fraction of this material can even become gravitationally unbound and might escape from the system (see Sect. 4.4). This angular momentum transport - and also the fact that asymmetric systems already have a smaller angular momentum in the pre-merging quasiequilibrium stage - leads to smaller spin parameters $a_{\text {remnant }}$ of the remnant in asymmetric systems (see Table 1). In some systems, this
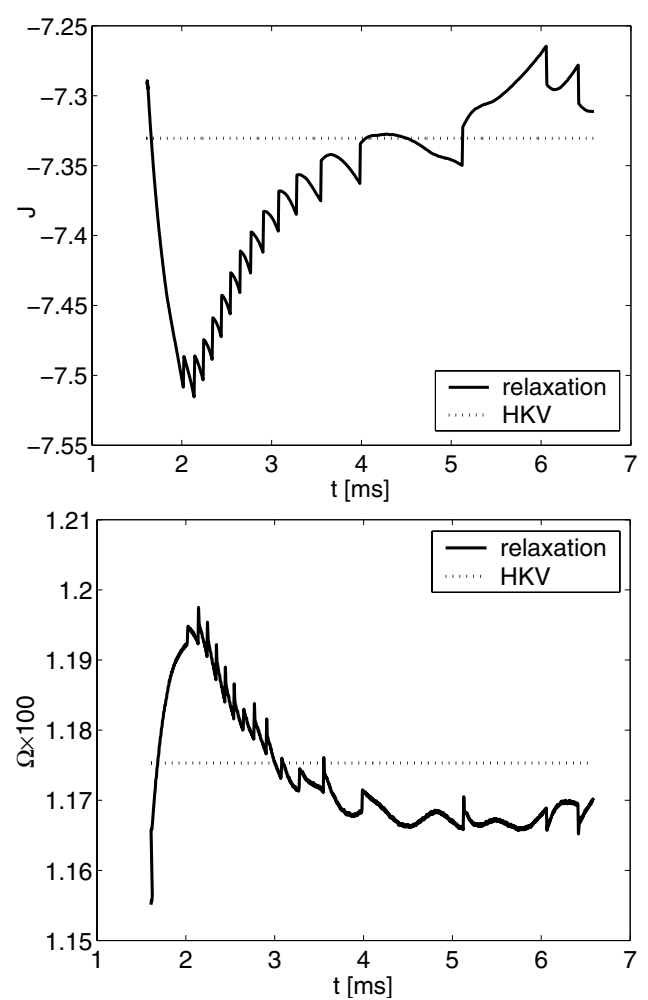

Fig. 4. Comparison of the angular momentum (top) and the angular velocity (bottom) from our relaxation scheme with results based on the helical killing vector (HKV) method. Both quantities are given in geometrical units $\left(G=c=M_{\odot}=1\right)$.

angular momentum difference leads to an immediate collapse to a BH after the merger (model LS1216) whereas the symmetric model (LS1414) with the same EoS and spin state collapses not until about $10 \mathrm{~ms}$ after merging. During the postmerger evolution, strong radial and non-axisymmetric oscillations of the central remnant periodically lead to the formation of smaller, secondary spiral arms and to successive ejection of material into the torus (see Fig. 5).

In contrast, in case of a mass ratio near unity as, e.g., in models S1414 or S138142, the two partners smoothly plunge into each other without any disruption and without the formation of a primary spiral arm. The resulting central object has a twin-core structure formed from the two original cores (Fig. 5, panel a). They are kept mostly intact during merging and lead to a rotating bar-like structure in the remnant center, which drives the formation of secondary spiral arms after the dynamical merger phase and, which leads to ejection of material into the torus. The bar-shaped core slowly transforms during the later evolution into a rotationally flattened high-density core. The mixing of material between the two initial NSs in the remnant is mainly taking place at the turbulent contact interface (see Fig. 6). Since the primary spiral arm is absent, a much smaller torus results after merging. Note, however, that primary spiral arms and large torus masses may still emerge if the initial NS spin setup is favourable, e.g. in case of corotating binaries as in model S1414co.

\subsubsection{Different neutron star spins}

The NS spins determine the velocity jump across the contact interface at merging. This velocity discontinuity leads to a Kelvin-Helmholtz (KH)-unstable vortex sheet between the two merging NSs and to shock heating (and heating by 


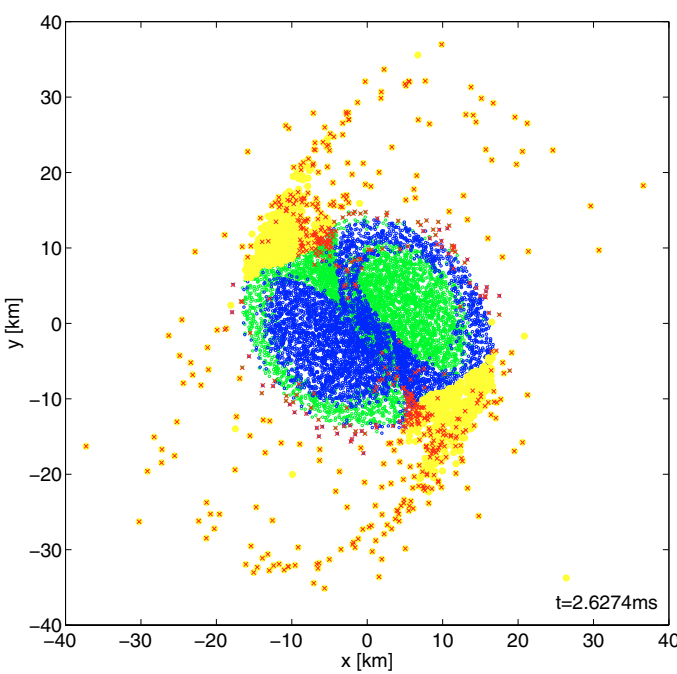

(a)
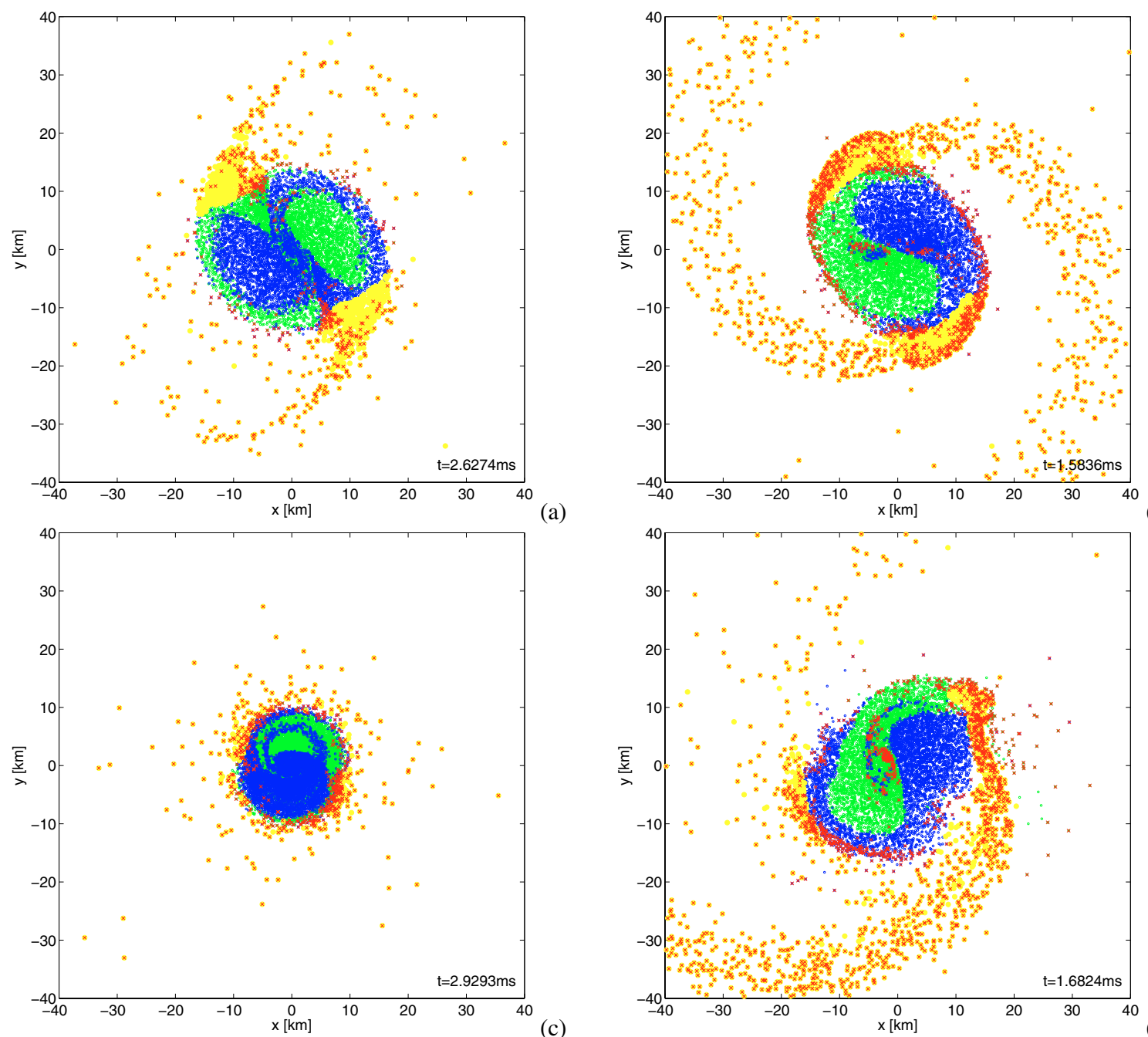

Fig. 5. Characteristic snapshots of four representative models. Plotted is every 10th SPH particle around the equatorial plane. The matter of the two NSs is color-coded by green and blue particles. Yellow circles represent particles which currently fulfill the torus criterion of Eq. (1) whereas particles ultimately ending up in the high-angular-momentum torus are plotted in red. By definition, the yellow and red particles coincide at the end of the run. Panel a) shows the development of secondary spiral arms in model S1414. Panel b) shows the large, primary spiral arms in model S1414co. The two clumps of red particles at the remnant surface are going to form secondary spiral arms which transport these particles into the torus. Panel c) shows a postmerger snapshot of model LS1414. The merger remnant in much more compact and no secondary spiral arms are formed. As a consequence, the torus is much smaller and develops only on a longer timescale. In panel d), we see the formation of the large primary spiral arm in model S1216. Similar to panel b), a secondary spiral arm begins to form in the area at the remnant surface where the red particles cluster.
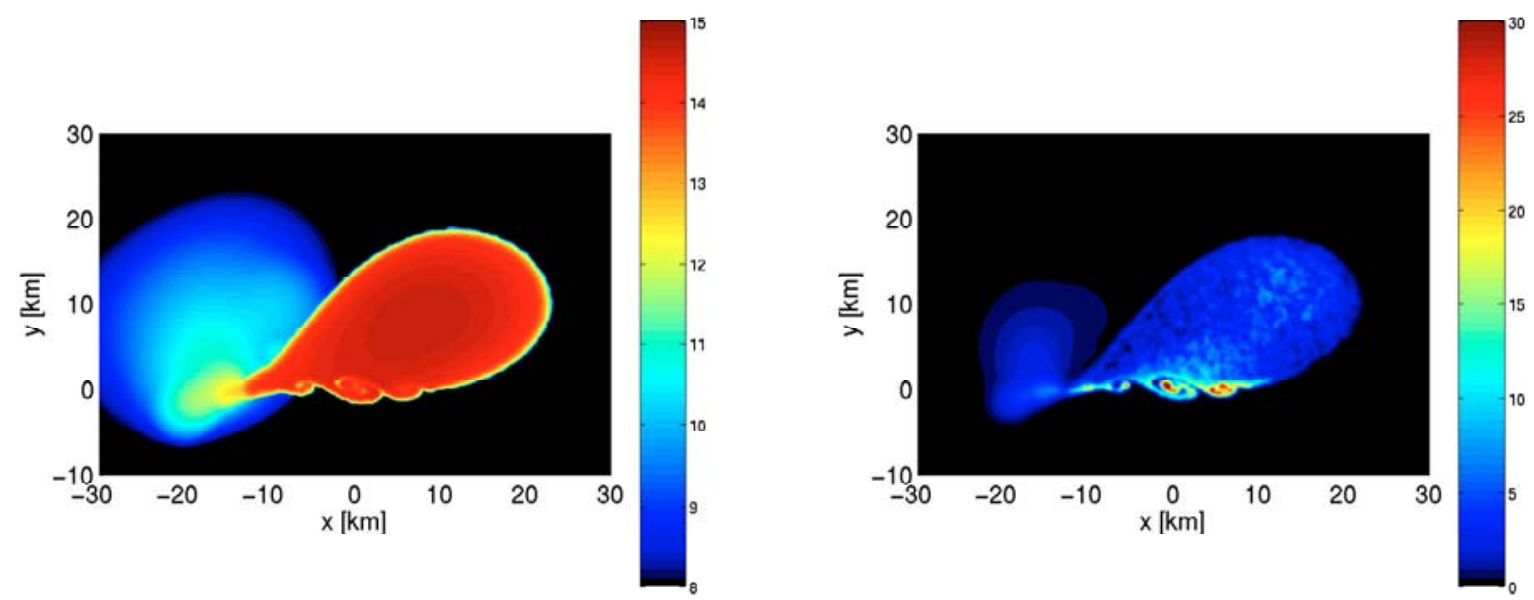

Fig. 6. Visualisation of the Kelvin-Helmholtz-unstable shear interface and of the associated shock-heating and viscous shear heating during merging of the two NSs in model S1414 at $t \simeq 0$. Plotted is the density (left; logarithmic scale, in $\mathrm{g} / \mathrm{cm}^{3}$ ) and temperature (right; linear scale, in MeV) distribution of the matter originating from one of the NSs of this model. 
numerical viscosity) along the contact interface. In models with a large velocity jump as in irrotating or even counterrotating models, we indeed see large $\mathrm{KH}$-vortices and strong heating (Fig. 6). In contrast, in corotating models like S1414co, there is only a small velocity jump which does not result from the spinning NSs but from the Coriolis force deflecting the infalling gas in the tidal tips into azimuthal direction shortly before merging. Consequently, $\mathrm{KH}$-vortices still develop, but on a longer timescale and with reduced shock heating and viscous heating.

A larger velocity jump and thus a stronger shear flow at the interface is also associated with stronger non-axisymmetric deformation of the central remnant. This can be seen, e.g., by considering the normalized $l=m=2$-mode amplitude $q_{2}^{2} / q_{0}^{0}=\int \rho^{*}\left(x^{2}-y^{2}\right) \mathrm{d}^{3} x / \int \rho^{*}\left(x^{2}+y^{2}+z^{2}\right) \mathrm{d}^{3} x$ which is an oscillating function whose amplitude can be considered as a measure for the deviation from non-axisymmetry. In Fig. 7, we see that the non-axisymmetry, as measured by the above quantity, is significantly enhanced at later times in the counterrotating model S1414ct compared to the irrotational (S1414) and corrotational (S1414co) model, respectively. The same is true for, e.g., S1216ct compared to S1216co. A direct consequence are differences in the strength of the postmerger gravitational wave signal, which we calculate from the second derivative of the quadrupole of the matter distribution using the common quadrupole formula (see Oechslin \& Janka 2007).

The NS spin setup also determines the total amount of angular momentum in the system and thus directly influences the large-scale structure of the merger remnant, the torus mass (Sect. 4.3), and also the amount of mass ejected from the system (Sect. 4.4). In corotating models, the total angular momentum right after merging is larger by about $8 \%$ compared to the irrotational case with the consequence that two primary spiral arms form during merging, carrying this additional angular momentum into a massive high-angular-momentum torus. The central remnant is left behind with a similar angular momentum content as in the irrotating case (see the parameter $a_{\text {remnant }}$ in Table 1). In the opposite, i.e. counterrotating case, the $8 \%$ reduction of the total angular momentum results in an even smaller torus and a more compact remnant. A spin configuration of the "tilted" type as e.g. in model S1414t1 (large initial NS spin in $y$-direction) leads to a remnant with a rotation axis parallel to the total angular momentum direction (including NS spins) but inclined relative to the orbital angular momentum of the initial binary. It also results in a diffuse, puffed-up halo with no welldefined symmetry.

\subsubsection{Equation of state}

In this section, we investigate how the characteristic features of our EoSs influence the merger dynamics and outcome. The supernuclear behaviour of the EoS determines the compactness of the initial NSs (see Sect. 2.2 and Fig. 2). In systems with larger NSs the plunge and merging phase sets in earlier, i.e. at a larger orbital separation, and thus, less energy and angular momentum is radiated away in such models. For instance, in S1414 about $7 \%$ of the total angular momentum is lost while in LS1414 about $12 \%$ and in A151414 about 15\% is radiated away during inspiral. Thus, besides the spin setup, also the NS compactness has a large influence on the angular momentum retained in the system right after merging. The corresponding spin parameter $a_{\text {system }}$ is listed in Table 1 .

The supernuclear behaviour of the EoS also crucially affects the structure of the merger remnant and its collapse time. For
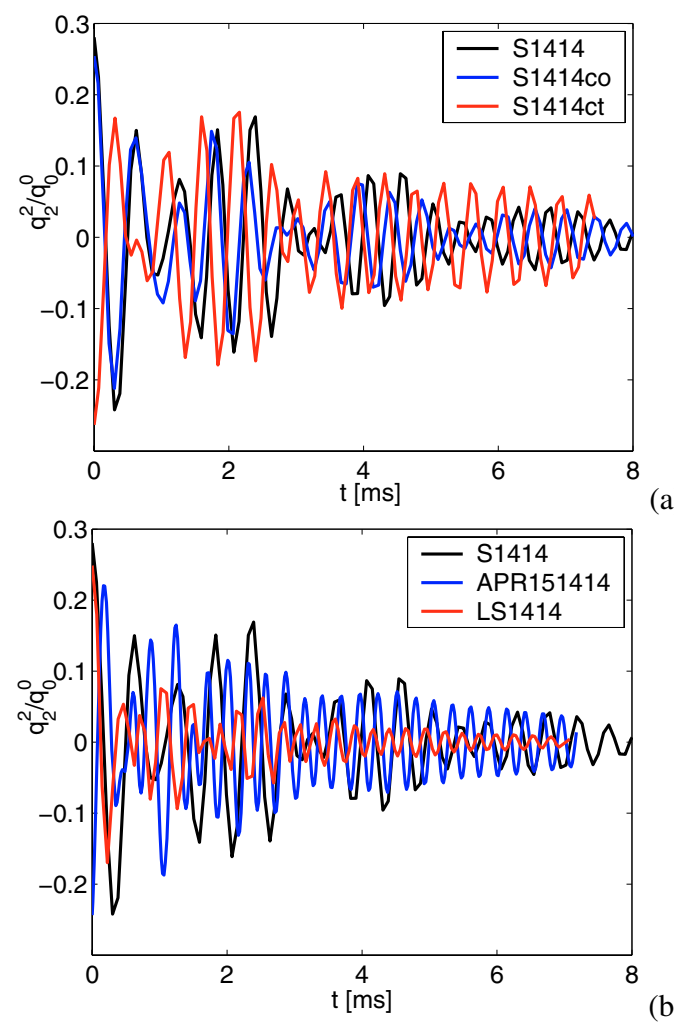

(a)

Fig. 7. a) Plotted is the normalized $l=m=2$-mode amplitude of the remnant rest mass distribution as defined in Sect. 4.1.2 for models S1414, S1414co and S1414ct. The evolution of the amplitude, which is a measure for a deviation from axisymmetry, is significantly larger at later times in the counterrotating model than in the other two cases. b) The same mode amplitude, but for the 1.4-1.4 $M_{\odot}$-models with the Shen-, LS- and APR-EoSs. The strength of non-axisymmetry increases with the stiffness of the supernuclear EoS. Note that the normalized $l=m=2$-mode is not sensitive to the compactness of the remnant.

instance, the remnants of the LS-EoS models both collapse to BHs shortly after merger, either immediately (LS1216) or delayed (LS1414), while in the corresponding Shen-EoS model S1414 and APR-EoS models A151414 and A21414, no sign of a collapse can be seen within the simulation time. The collapse time has a direct influence on the torus mass, since secondary spiral arms, in which angular momentum transport to matter at larger radii takes place, only form if the central remnant does not collapse immediately.

The EoS stiffness in the supernuclear regime also affects the non-axisymmetry of the merger remnant. As suggested by earlier simulations of bar-mode instabilities in NSs, a higher stiffness favours larger and longer-lasting non-axisymmetric oscillations (e.g. Houser \& Centrella 1996; Shibata et al. 2003). This mainly affects the strength of the gravitational wave signal, but gravitational torques transporting angular momentum to larger radii are also amplified (see Sect. 4.3).

The subnuclear EoS influences the merger and postmerger dynamics only weakly. A comparison of models P1315 (ideal gas EoS) and S1315 (Shen-EoS) yields similar remnant structures and torus masses (see Fig. 12) but also similar density profiles in the low-density regime outside the remnant where the thermal contributions to the pressure become important. We cannot find a clear reason for the latter observation. It might be caused by the fact that the coefficient $\partial p /\left.\partial \epsilon\right|_{\rho}$ is much larger in the ideal-gas EoS at densities below about $5 \times 10^{13} \mathrm{~g} / \mathrm{cm}^{3}$ (see Fig. 1). This might compensate the initially much lower pressure 
in that density regime, once the energy density starts to increase in that regime due to heating.

The additional thermal pressure provided by a higher value of $\Gamma_{\text {th }}$ in the APR-EoS leads to slightly $(\sim 10 \%)$ lower central densities and a less compact structure of the merger remnant in model A21414 $\left(\Gamma_{\text {th }}=2\right)$ compared to model A151414 $\left(\Gamma_{\text {th }}=1.5\right)$. The same effect can be observed as a result of the thermal pressure and energy in the Shen case compared to the cold-Shen models. The additional thermal pressure becomes more noticeable at lower densities. It leads to more extended (but less massive, see Sect. 4.3) low-density halos around the merger remnants in models A21414, S1315, and S1216 ("hot models") compared to their analogues A151414, C1315, and C1216 ("cold models"), respectively (also see Sect. 4.2).

\subsection{Thermal evolution of the finite temperature EoS-models}

The initial NS models are set up with a temperature of $0.1 \mathrm{MeV}$, which is the lowest value tabulated in the EoS tables. During inspiral, matter is heated up in the center at most to a few MeV due to numerical viscosity, which however has no dynamical consequences because thermal effects due to temperatures of less than a few $\mathrm{MeV}$ yield a negligeable pressure contribution at the high densities in the NS interior. There are three locations where significant heating takes place during merging and the postmerger evolution: At the Kelvin-Helmholtz(KH)-unstable collision interface between the two NSs, at the remnant surface, and in the torus itself (see Fig. 8).

The contact interface is shock heated by the clash of the two NSs and by the turbulent motion with viscous dissipation due to numerical viscosity in the KH-vortices. Shocks also form at the surface of the non-axisymmetric, rapidly rotating merger remnant when it collides with the low-density surrounding halo matter which has been ripped off the NS surfaces along the shear interface at merging (see the temperature distribution shown in Fig. 8, panels $\mathrm{b}$ and $\mathrm{c}$ and Fig. 10). Finally, heating takes place in the torus itself due to collision of the dense spiral arms with each other or with ambient torus matter.

The strength of shock and viscous heating can be shown by considering the thermal contribution $\epsilon_{\text {therm }}=\epsilon\left(\rho, T, Y_{\mathrm{e}}\right)-$ $\epsilon\left(\rho, T=0, Y_{\mathrm{e}}\right)$ to the internal energy $\epsilon$. In the upper panel of Fig. 9, we show for the three symmetric models S1414co, S1414 and $\mathrm{S} 1414 \mathrm{ct}$ the ratio $\epsilon_{\text {therm }} / \epsilon\left(\rho, T, Y_{\mathrm{e}}\right)$, which is zero where no shock heating is experienced and which tends to unity where the degeneracy energy $\epsilon\left(\rho, T=0, Y_{\mathrm{e}}\right)$ is negligible compared to the thermal component $\epsilon_{\text {therm }}$. We clearly see that the thermal contribution is largest in the counterrotating model and still larger in the irrotating model than in the corotating case because of the more extreme velocity jump at the contact interface. In addition, the counterrotating model yields a larger non-axisymmetry of the remnant and thus also leads to stronger shock heating at the remnant surface (see Fig. 11). We also note that the torus material in the counterrotating model S1414ct is hotter because it originates from the shock-heated remnant surface rather than being ejected in unshocked spiral arms as in models S1414co and S1414. In these models, the torus material is initially cool but gets gradually heated up by collisions of the spiral arms.

We generally obtain higher temperatures in the LS-EoS models than in the Shen-EoS ones. These higher temperatures are accompanied by the higher densities in the more compact merger remnants of the LS-models, which suggests that the temperature difference is mainly caused by adiabatic compression of the remnant interior.

\subsection{Torus mass estimates}

Prior to merging only very few fluid elements, e.g. in corotating models, have a specific angular momentum which is large enough to fulfill the torus criterion of Eq. (1). But angular momentum is transported from the central remnant outward to larger radii by gravitational torques, which are generated by the forming non-axisymmetric merger remnant during the merging and early postmerger evolution. In models where a primary spiral arm is present, this transport process is particularly efficient, and a rapid rise of the torus mass occurs during the violent collision phase (see Figs. 12 and 5, panel d). We find that a large part of the future torus matter originates from the primary spiral arm. Secondary spiral arms during the further evolution also contribute, although on a lower efficiency level.

Test calculations with a physical setup similar to that of model S1214 but with different particle numbers reveal that the torus mass is not very sensitive w.r.t. the numerical resolution. The model using $~ 120000$ particles differs from the one using $\sim 280000$ particles by less than $5 \%$ in torus mass.

With NS spins and EoS fixed, we find an increase of the torus mass when the mass ratio drops below unity, while the total mass of the system has only little influence (see Fig. 2 in Oechslin \& Janka 2006). The torus mass in case of the Shen EoS rises rapidly from about $0.04 M_{\odot}$ at $q=1$ to about $0.2 M_{\odot}$ at $q \simeq 0.8$ and then roughly saturates for even smaller $q$-values. For a $q$-value of 0.55 we find a torus mass of about $0.25 M_{\odot}$.

When the EoS and the mass ratio are fixed, the torus mass increases with the total amount of angular momentum available in the system. Thus, corotating models yield a larger torus mass than irrotating models, counterrotating smaller ones. Remarkably, models with a different spin setup, but the same total angular momentum, yield similar torus masses. This is the case for models S1414co (corotating) and S1414t2 (tilted NS spins which sum up to a spin contribution aligned with the orbital spin), and roughly also for models S1414 (no spins) and S1414o (oppositely oriented spins). This suggests that the dependence of the torus mass on the NS spin setup can be approximately reduced to a single parameter, the total angular momentum in the system (see Fig. 13).

Finally, the EoS influences the torus mass in various ways during the different phases of the merging and postmerging evolution. EoSs which yield less compact NSs like the Shen-EoS lead to lower angular momentum loss during inspiral (Sect. 4.1.3), thus favouring larger tori. Moreover, at the time of plunging, more angular momentum is transferred to the outermost NS matter sitting opposite to the merger interface in case of larger NSs. This explains the steep increase of the torus mass in the Shen-EoS models shortly after merging (see Fig. 12). The corresponding models with the same mass ratio and spin setup but using the LS-EoS and the APR-EoS show a slow and continuous growth of the torus mass setting in only about $1 \mathrm{~ms}$ after merging.

During the postmerging evolution a second effect becomes important. The more compact remnants allow for a more efficient angular momentum transport through tidal torques from the central remnant to larger radii. This effect is visible in case of model LS1414 and of the two APR-EoS models. In the APR-cases it appears even more strongly due to the larger nonaxisymmetry (see Fig. 7 panel b) of the remnant. A part of this growth, however, is likely to be caused by numerical viscosity. This becomes clear at late times ( $\geq 5 \mathrm{~ms}$ ) in model LS1414, when the remnant has already settled to a nearly axisymmetric state, but the torus mass is still growing, although at a lower rate. 
(a)

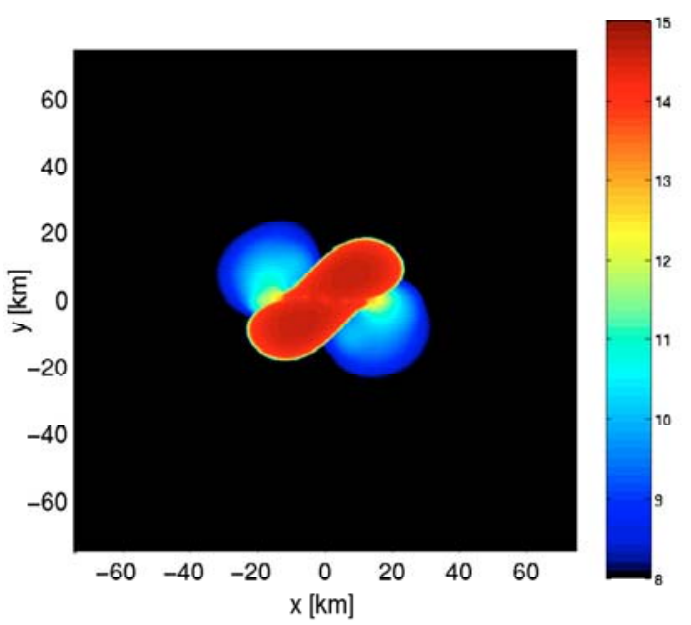

(b)
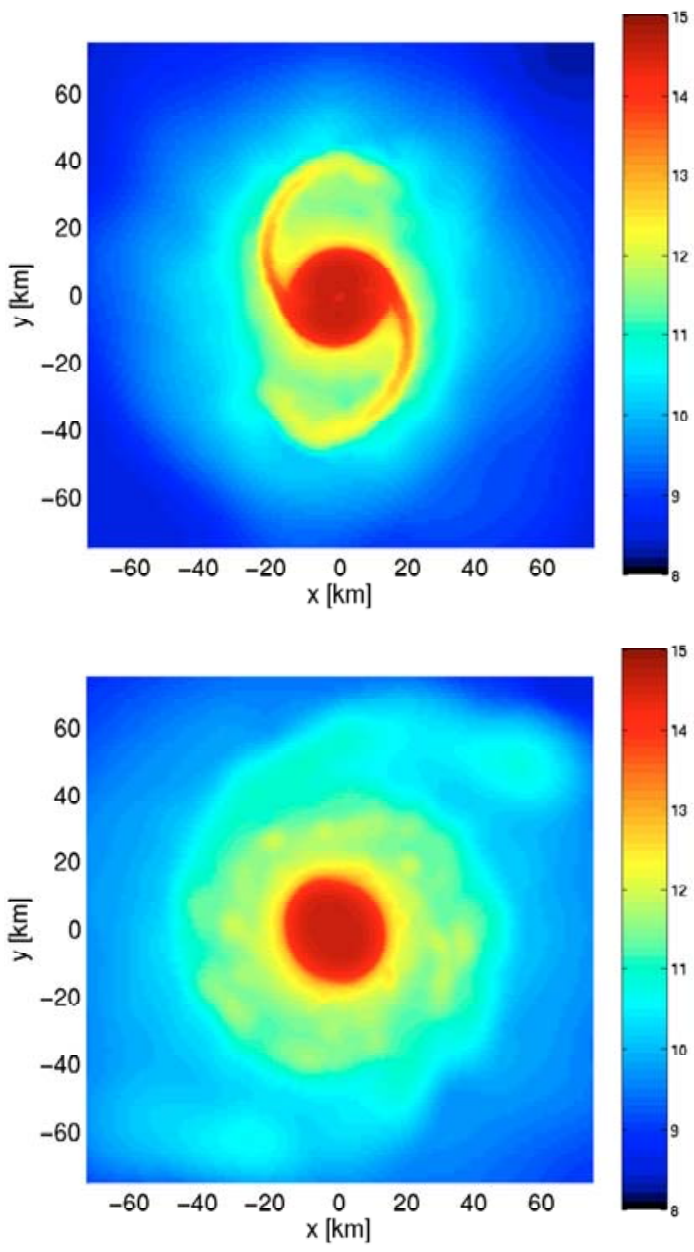
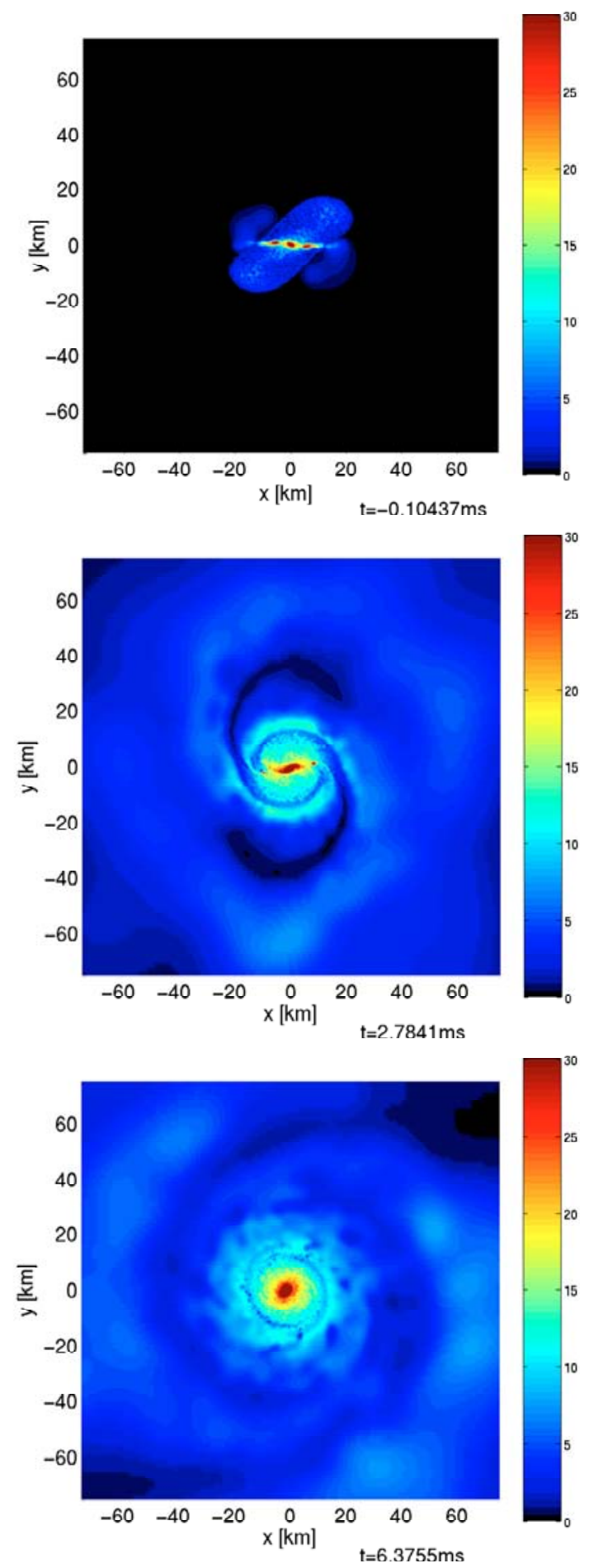

Fig. 8. Density (left; logarithmic scale, in $\mathrm{g} / \mathrm{cm}^{3}$ ) and temperature (right; linear scale, in MeV) distribution in the orbital plane for model S1414 at the times given below the right panels. A primary spiral arm is missing but a pair of secondary spiral arms forms shortly after merging. Maximum temperatures are reached in the hot and KH-unstable vortex sheet between the two merging NSs (panel a), right). Visible are also the shock-heated layers at the surface of the merger remnant and along the leading edge of the spiral arms which plough through the less dense torus material (panel b), right). Panel c) shows a later stage where the dense and hot merger remnant is surrounded by a "warm" torus.

In models where the thermal pressure is neglected (C1216 and $\mathrm{C} 1315)$ or reduced (A151414), we observe larger torus masses compared to the corresponding models S1216, S1315 and A21414, respectively. This difference develops mainly during the early postmerger phase, which suggests that the reason may be found in the different efficiency of the angular momentum transport to the torus right after the actual merging event. This claim is supported by the fact that in the cold models, the matter density is higher close to the surface of the newly formed remnant than in the hot models due to the lack of thermal 

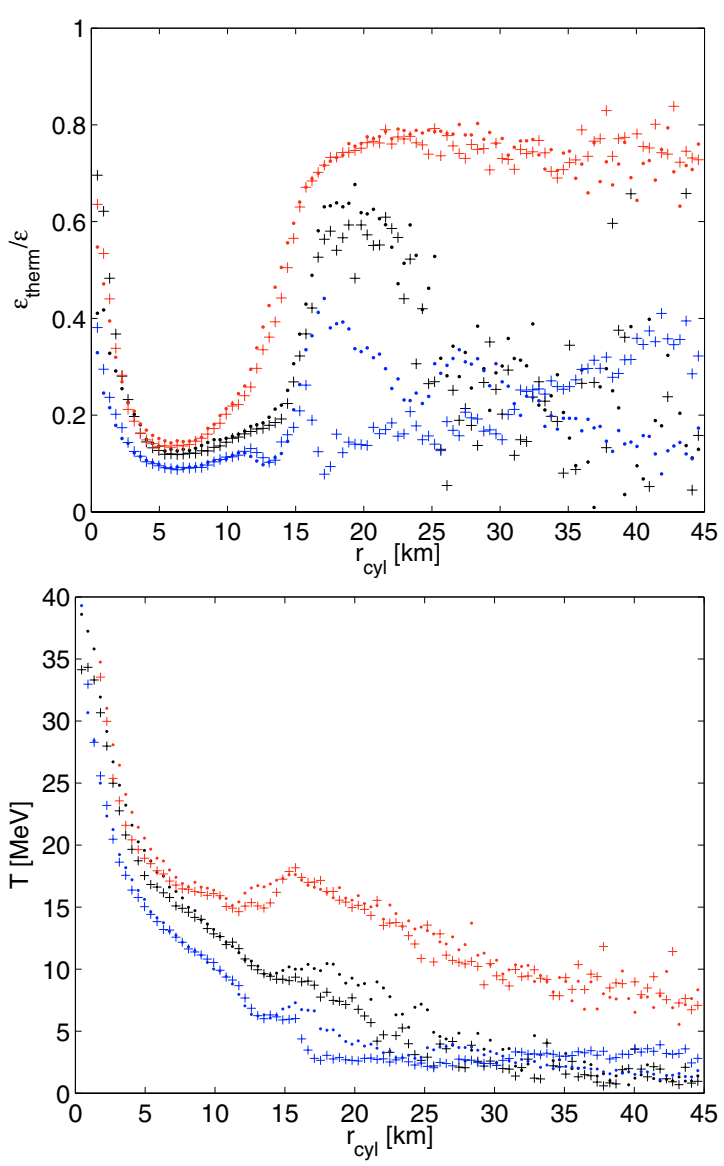

Fig. 9. Thermal energy contribution $\epsilon_{\text {therm }} / \epsilon=1-\epsilon(\rho, T=0$, $\left.Y_{\mathrm{e}}\right) / \epsilon\left(\rho, T, Y_{\mathrm{e}}\right)$ relative to the total internal energy (top panel) and the corresponding temperatures as given by the EoS (bottom panel) versus the cylindrical radius $r_{\text {cyl }}=\sqrt{x^{2}+y^{2}}$ at around $5 \mathrm{~ms}$ (crosses) and $7 \mathrm{~ms}$ (dots) after merging for three different models, S1414 (irrotating; in black), S1414co (corotating; in blue) and S1414ct (counterrotating; in red). The plotted values are obtained by angle-averaging the matter distribution in the orbital plane.

pressure. Thus, more matter is residing in the areas where gravitational torques are strongest and more angular momentum and ultimately mass is transferred outwards in the cold models.

\subsection{Estimated ejected mass}

We estimate the amount of ejected, i.e. gravitationally unbound, matter by considering for each fluid particle the quantity (Oechslin et al. 2002)

$e_{\mathrm{tot}}=v^{i} \hat{u}_{i}+\frac{\epsilon}{u^{0}}+\frac{1}{u^{0}}-1$

which is conserved (in the comoving frame) if pressure forces are small compared to gravitational forces and if the metric is stationary. These conditions are well fulfilled for cold gas in an axisymmetric, stationary torus, but are certainly only approximately realized in our dynamical models. The quantity $e_{\text {tot }}$ asymptotes to the Newtonian expression for the total energy $v^{2} / 2+\epsilon-\phi, \phi$ being the Newtonian gravitational potential. For a particle to be gravitationally unbound, this energy has to be positive and we therefore use the criterion $e_{\text {tot }}>0$ for matter to be considered as ejected from the merger site.

We find that ejected matter basically comes from two locations, the tip of the spiral arms in models where such arms form, and the collision interface of the merging NSs, where matter is ejected perpendicular to the orbital plane (see Fig. 14). Thus, we expect the ejected matter to consist of a hot/high-entropy component from the shock-heated collision interface and of a cold/low-entropy component from the tail of the primary spiral arm(s). In Fig. 15, we show the distribution of the ejecta for the two models S1414 and S1216 in the density-temperature plane. We have color-coded the amount of ejecta mass per unit of density and temperature. Clearly visible and well-separated are a hot and a cold component. The latter is only present in the asymmetric model S1216 where the cold component originates from the tip of the spiral arm, while the hot component comes from the shock-heated merger interface.

Generally, we find ejecta masses from about $10^{-3} M_{\odot}$ to a few $10^{-2} M_{\odot}$ (Table 1 ). The mass grows with the asymmetry of the system. It also becomes larger, unlike the torus mass, for counterrotating systems where the stronger shear flow accelerates more hot matter at the collision interface to high velocities without giving it a high angular momentum. Since the amount of ejected matter still increases at the end of our simulations, we cannot provide precise numbers for the ejecta masses.

\subsection{Comparison with results of other works}

Model A21414 can be compared with model APR1414 of Shibata \& Taniguchi (2006). We obtain the same remnant object, a hypermassive NS, and we find good agreement in the global evolution, with somewhat higher values for the central lapse function, i.e., smaller central densities in our model. The reason for this difference might be the smaller angular momentum loss in GWs, which is slightly underestimated by our backreaction scheme compared to the simulation of Shibata \& Taniguchi (2006). In our model $\sim 7 \%$ of the initial angular momentum are radiated away during the last two ms before merging, while it is $\sim 11 \%$ in the calculation of Shibata \& Taniguchi (2006). These authors estimate a torus mass for their model of about $0.02 M_{\odot}$, which is slightly smaller than our value $\left(0.03-0.04 M_{\odot}\right)$. We expect the reason to be again the smaller angular momentum loss by GWs in our model and therefore the higher angular momentum of our postmerger configuration. It is currently unclear to what degree this difference in torus masses could also have numerical origin (e.g. SPH vs. grid-based calculation, resolution issues, numerical viscosity) instead of being caused by the CFC approximation. It is certainly desirable to attempt direct comparative calculations with different codes and treatments of GR in the future. Considering an asymmetric model with the APR-EoS, Shibata \& Taniguchi (2006) found a much lower sensitivity of the torus mass to the mass ratio than we did for our models with the Shen-EoS. The reason might be the much smaller NS radii in case of the APR-EoS. We have seen in all our models with small NS radii (LS1414, LS1216, A151414, A21414 and in a test case with a mass ratio of $q=0.875$ using the APR-EoS) that the growth of the torus mass during merging is very small compared to the growth setting in $\sim 1 \mathrm{~ms}$ after merging. Therefore, the dominant process for torus formation in these cases is the angular momentum transport in the non-axisymmetric postmerger remnant (see Sect. 4.3). But at that stage, mass-ratio effects are much smaller that at the stage of merging. This might explain the much smaller mass ratio dependence observed by Shibata \& Taniguchi (2006) in their APR-models.

Our maximum temperatures at the center of the remnants are generally considerably higher than those obtained for similar models in Newtonian simulations (Ruffert \& Janka 2001; 

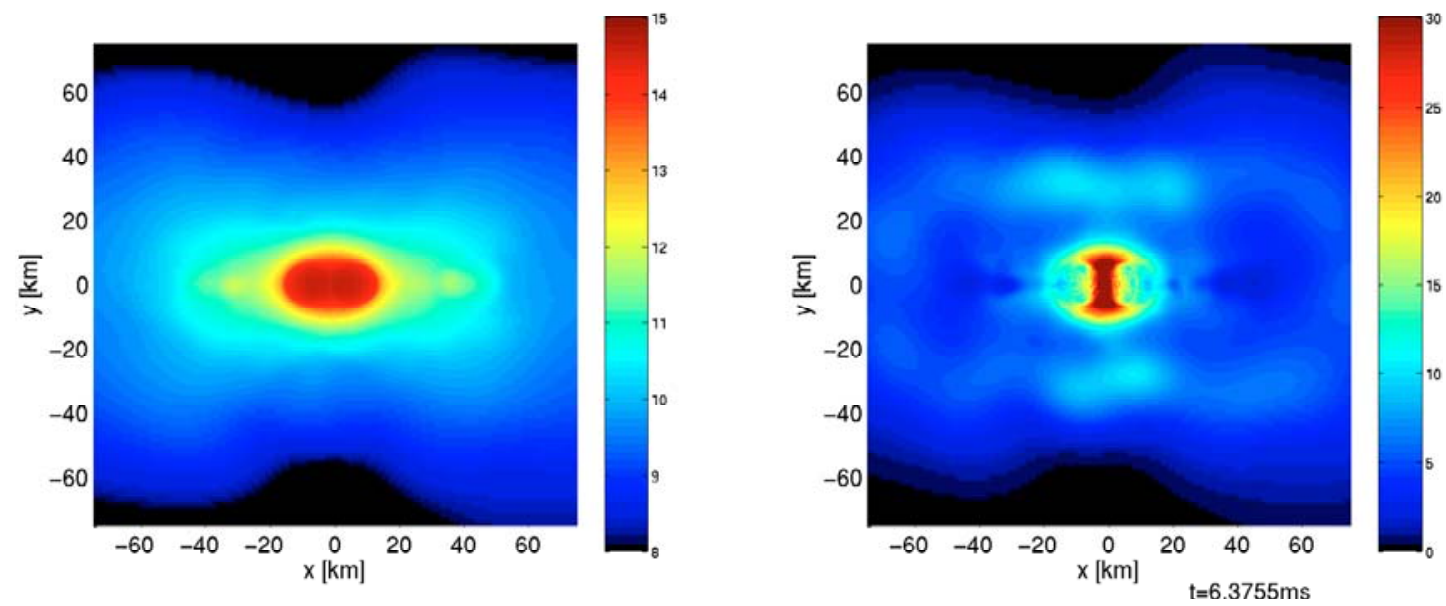

Fig. 10. Same situation as Fig. 8, lower panels, but perpendicular to the orbital plane.
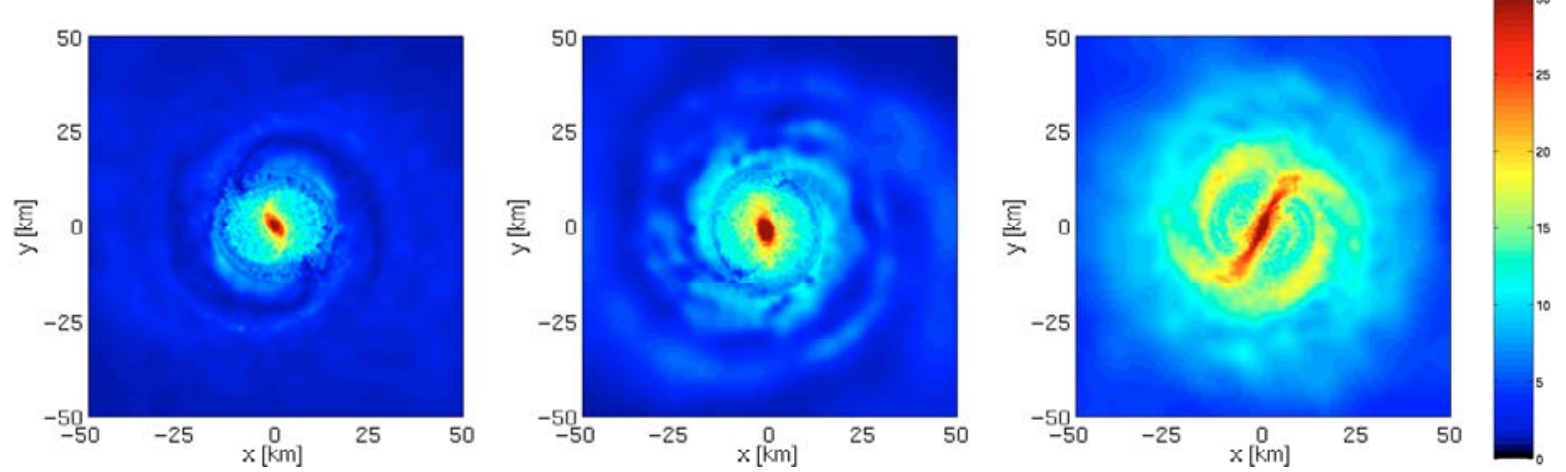

Fig. 11. Temperature distribution in the equatorial plane of the remnant and the torus shown for models S1414co (corotating; left), S1414 (irrotating; middle) and S1414ct (counterrotating; right) at $\sim 5 \mathrm{~ms}$. Notice the different temperatures at the collision interface of the two NSs and at the remnant surface.

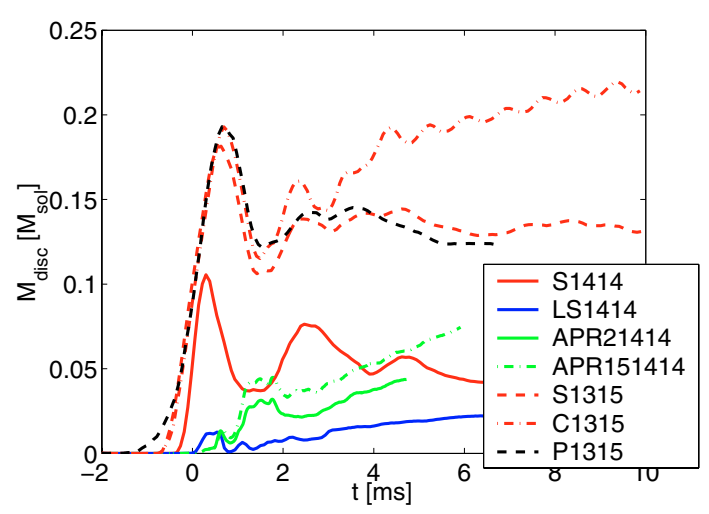

Fig. 12. The evolution of the torus mass for models with different EoSs. Models with large initial NSs, as in case of the Shen-EoS and the idealgas EoS (in red and black, respectively), show a rapid rise of the torus mass already during the merging, while models with small initial NSs (in blue and green, respectively) show a slow but continuous increase of the torus mass setting in about $1 \mathrm{~ms}$ after merging. Models S1315 (red dashed) and P1315 (black dashed) illustrate the similar torus mass evolution obtained with the Shen EoS and the ideal-gas EoS. Finally, models A151414 and C1315 (to be compared with A21414 and S1315, respectively) illustrate the larger torus mass obtained using EoSs with reduced thermal pressure contributions. Note: In contrast to Oechslin \& Janka (2006), we have included the non-compact $K_{i j} K^{i j}$ term in the central remnant gravitational mass for calculating the torus mass here. Due to this, we obtain slightly smaller values in the present work.

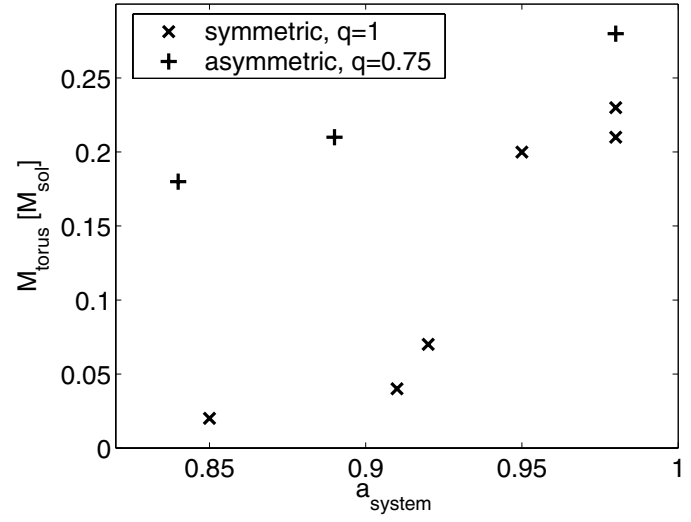

Fig. 13. Estimated torus masses versus total spin parameter $a_{\text {system }}$ of the systems measured immediately after merging. Plotted are two series of models with mass ratio $q=1$ (crosses) and $q=0.75$ (plus signs), respectively. In all models, the Shen-EoS was employed.

Rosswog \& Davies 2002), both with the Shen-EoS and the LS-EoS (e.g. S1414 compared to model C in Rosswog \& Davies 2002 or model A64 in Ruffert \& Janka 2001). This can be explained by the stronger gravity in our relativistic models. The consequently enhanced adiabatic compression of the matter in the remnant leads to higher temperatures.

We also find a smaller torus mass in our model LS1414 compared to model A64 in Ruffert \& Janka (2001) which has been 

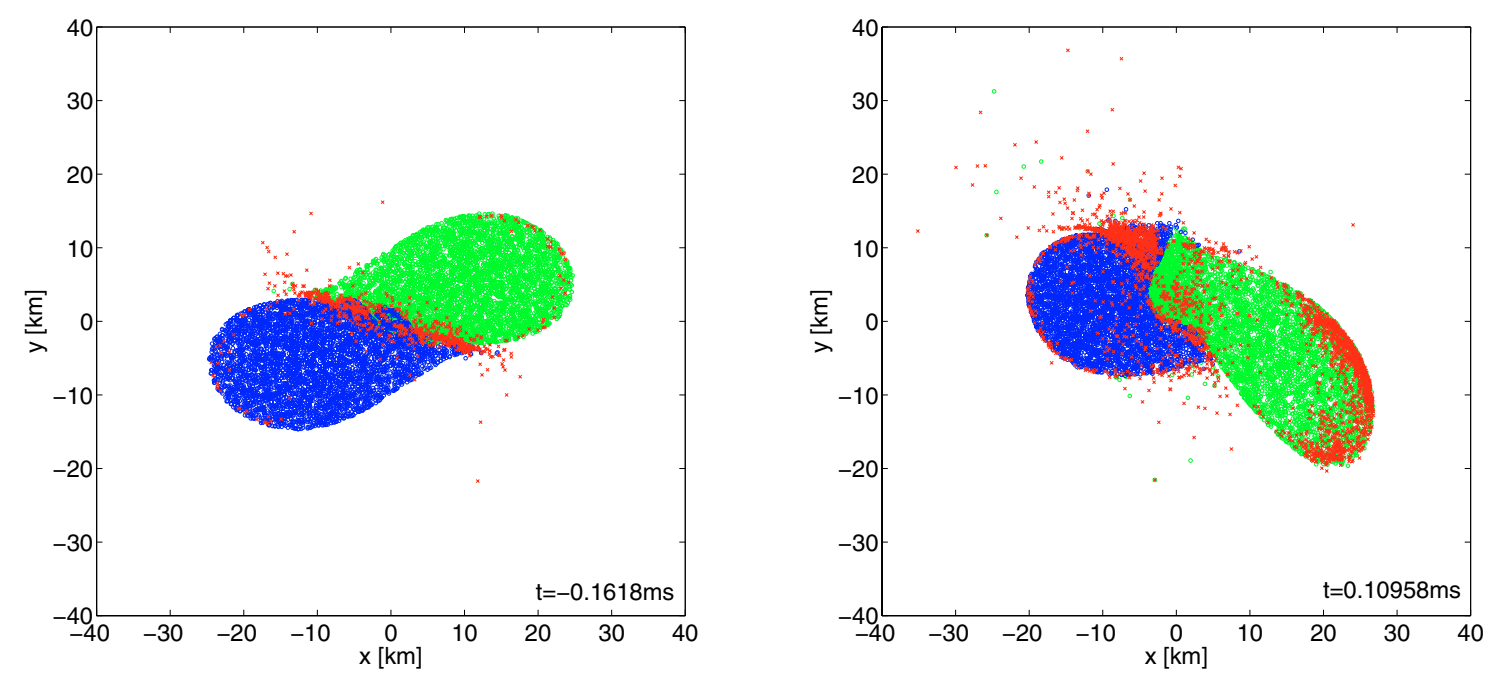

Fig. 14. Snapshots at the moment of merging of a symmetric (S1414; left) and an asymmetric (S1216; right) model. The particles color-coded in red become gravitationally unbound during the postmerger evolution. We can identify two sources of ejected matter, the merger interface and the tip of the primary spiral arm (if present). Note that the ejected matter is over-emphasized in this plot since we plotted every second ejecta particle whereas for the remaining matter only every 10th particle near the equatorial plane is plotted. The red particles at the contact interface are therefore squeezed out perpendicular to the orbital plane.
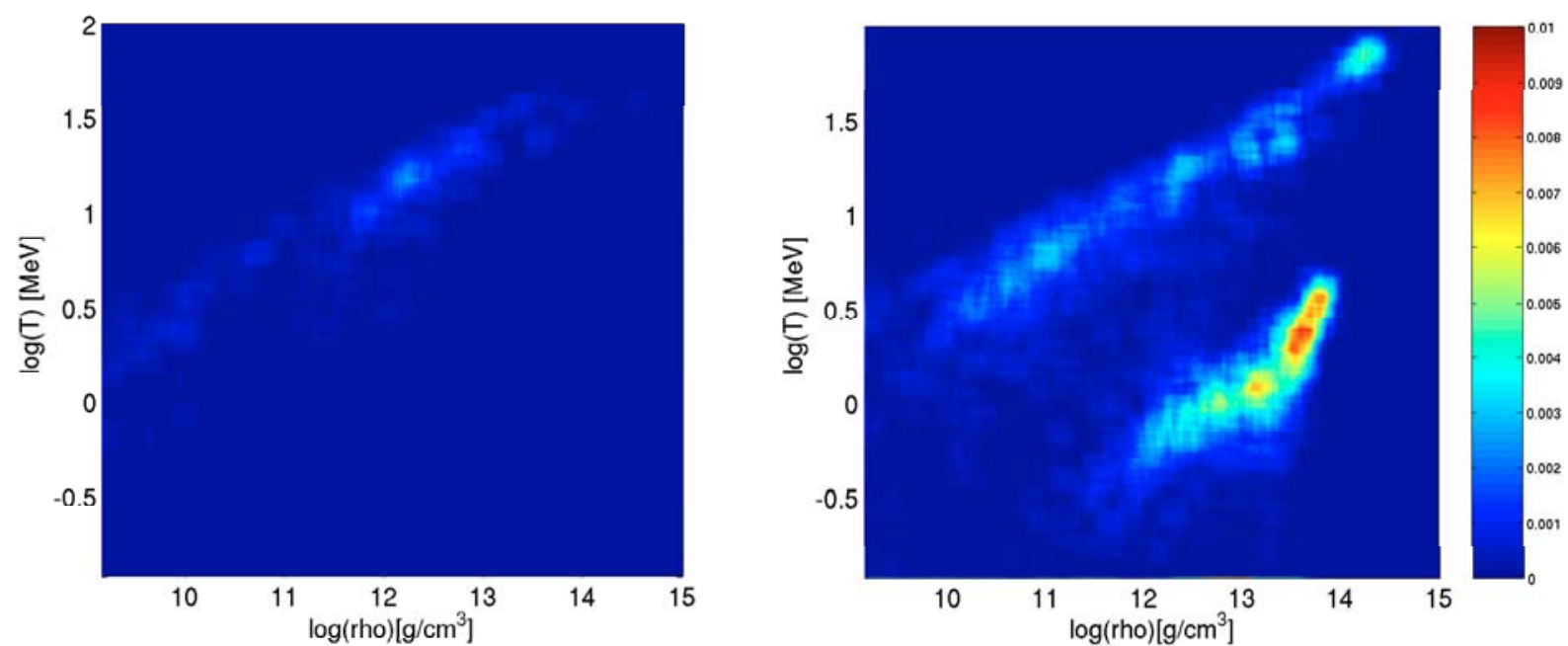

Fig. 15. Shown is the distribution of ejected matter in the density-temperature plane around $1 \mathrm{~ms}$ after merging for models S1414 (left) and S1216 (right). Color-coded is the amount of ejecta matter per unit of density and temperature. We can identify two contributions to the ejecta, a cold/lowentropy component and a hot/high-entropy one. The cold component is only present in model S1216, which suggests that this matter comes from the tip of the spiral arm, while the hot component that exists in both cases stems from the merger interface.

calculated with the same binary parameters and the EoS but using Newtonian gravity. Our calculations with the LS-EoS yield merger remnants which collapse BHs within a few ms, while the Newtonian calculation yields a stable merger remnant.

\section{Conclusions}

We have carried out a set of relativistic binary NS merger calculations starting from double NS binaries in quasiequilibrium, evolving them through merging until the formation of a hypermassive NS surrounded by a thick torus. We have considered two different physical, non-zero-temperature EoSs, the ShenEoS and the LS-EoS, an ideal-gas EoS with parameters adapted to match the Shen-EoS at very high densities and $T=0$, and the zero-temperature APR-EoS with an ideal-gas-like extension to mimic thermal effects as used by Shibata \& Taniguchi (2006). We have also considered different choices for the initial
NS masses and spins. The Einstein equations are solved in the conformally flat approximation.

We have seen that the merger dynamics and outcome depend crucially not only on the nuclear EoS, but also on the NS mass ratio and the NS spins. The total mass in the system was shown to be less critical. Our results can be summarized in the following way:

- The angular momentum in the system is determined by the NS spins but also by the size of the initial NSs. Corotation of the NSs increases the total spin by about $8 \%$ while counterrotation reduces it by about the same amount. In systems with large initial NSs, the final plunge and merging phase sets in earlier during inspiral such that up to $\sim 8 \%$ less angular momentum are radiated away compared to models with small NSs. We find a clear correlation between the angular momentum retained in the system and the torus mass. 
- The torus mass is very sensitive to the mass ratio, ranging from about $1-2 \%$ of the (baryonic) mass in symmetric systems up to about $9 \%$ of the system mass for a $q$-value of 0.55 .

- Merger remnants from asymmetric systems contain less angular momentum and are thus more compact and more likely to collapse earlier to a $\mathrm{BH}$ compared to their counterparts in symmetric systems.

- The EoS determines not only the size of the initial NSs but also influences the structure of the postmerger remnant and its collapse time to a BH. Models using the stiff ShenEoS or APR-EoS do not show any sign of a collapse until the end of the calculations at $\sim 10 \mathrm{~ms}$ after merging, while the soft LS-EoS leads to an immediate (model LS1216) or delayed (model LS1414) collapse of the remnant. The non-axisymmetry is largest in models using the APR-EoS, whereas the remnant in the LS-EoS model LS1414 quickly settles down to an axisymmetric state before collapsing a few ms later. This mainly affects the strength of the gravitational-wave signal, but angular momentum through gravitational torques are also amplified.

- The influence of the thermal pressure contribution of the EoS has been investigated by artificially setting the internal energy during the calculation to its value at $T=0$. While the influence of $T \neq 0$-effects is rather small in the high-density regime $(10 \%$ difference in the remnant central density), the thermal pressure becomes very important in the low-density postmerger torus. If thermal effects are neglected, postmerger tori are not only more compact but also more massive. This torus-mass difference develops in the early phase some ms after merging. The reason may be found in a higher matter density close to the surface of the newly formed compact remnant in the cold models due to the lack of thermal pressure. Therefore, more matter resides in regions where gravitational torques are strongest and more angular momentum and ultimately mass is transferred outwards in the cold models.

- Around $10^{-3}-10^{-2} M_{\odot}$ become gravitationally unbound. Two sources in the system, the merger interface and the tip of the primary spiral arm (only present in asymmetric systems), can be identified. Matter from the merger interface undergoes shock heating and is therefore ejected hot and entropyrich, whereas matter from the spiral arm(s) remains cold. The ejected mass increases with the asymmetry of the system but is also found to be sensitive to the spin state.

- Where direct comparison is possible, our results are qualitatively similar to those obtained by Shibata \& Taniguchi (2006). These authors estimate a torus mass of $\sim 0.02 M_{\odot}$ for their model APR1414 which is compatible with our value of $\sim 0.03-0.04 M_{\odot}$ (still growing at the end of the simulation) for our model A21414. Quantitative differences occur in the amount of angular momentum carried away be GWs ( $\sim 7 \%$ in our model A21414 compared to $\sim 11 \%$ in their model APR1414 during the last two ms before merging) with implications for the compactness of the merger remnants.

- We find generally higher temperatures and densities than those obtained in Newtonian calculations (Ruffert \& Janka 2001; Rosswog \& Davies 2002).

Our relativistic DNS merger simulations have extended previous work (e.g. Oechslin et al. 2004; Faber et al. 2004; Shibata et al. 2005; Shibata \& Taniguchi 2006) to the investigation of models with different NS masses, mass ratios, and spins, using microphysical EoSs with a fully consistent description of non-zero temperature effects in comparison to simplified EoS treatments.
Thermal effects are irrelevant in the inspiral phase and for determining the last evolutionary stages until the binary becomes unstable. During the final plunge and after the merging, however, the colliding stars heat up and the structure and post-merging evolution of the remnant depend on $T \neq 0$ effects, e.g. concerning the maximum density and compactness and the possibility of mass and angular momentum transfer from the remnant core to a low-density torus. While thermal effects tend to reduce the growth of such a torus during the evolution of the merger remnant, they significantly increase the mass ejection during the merging because hot matter from the collision interface of the two NSs can become gravitationally unbound.

In a subsequent paper we will evaluate the gravitationalwave signals from our mergers models, characteristic features of which can provide information about important EoS properties (Oechslin \& Janka 2007). More studies along two lines also appear highly desirable. On the one hand, a more detailed comparison between fully relativistic models and the presented simulations with conformally flat gravity is needed to assess the limitations of the approximative treatment in the merger context. We repeated some calculations found in another publication and obtained qualitative agreement. The remaining quantitative differences, however, could at least partly be caused by resolution differences or differences in the initial and boundary conditions, or by differences in the numerical viscosity of the schemes rather than the approach to GR. On the other hand, many of the observed differences between the models studied in this work could only be discussed in a descriptive way. Many more calculations with systematic variations of the initial conditions and employed equations of state are needed to work out dependences in detail and to develop a better understanding of the causal links between model input and results.

Acknowledgements. This work was supported by the SonderforschungsbereichTransregio 7 "Gravitational Wave Astronomy" and by the Sonderforschungsbereich 375 "Astro-Particle Physics" of the Deutsche Forschungsgemeinschaft. The computations were performed at the Rechenzentrum Garching and at the Max Planck Institute for Astrophysics.

\section{References}

Akmal, A., Pandharipande, V. R., \& Ravenhall, D. G. 1998, Phys. Rev. C, 58, 1804

Aloy, M. A., Janka, H.-T., \& Müller, E. 2005, A\&A, 436, 273

Ayal, S., Piran, T., Oechslin, R., Davies, M. B., \& Rosswog, S. 2001, ApJ, 550, 846

Bardeen, J. M., Press, W. H., \& Teukolsky, S. A. 1972, ApJ, 178, 347

Berger, E. et al. 2005, Nature, submitted [arXiv: astro-ph/0508115]

Bildsten, L., \& Cutler, C. 1992, ApJ, 400, 175

Blanchet, L., Damour, T., \& Schäfer, G. 1990, MNRAS, 242, 289

Blinnikov, S. I., Novikov, I. D., Perevodchikova, T. V., \& Polnarev, A. G. 1984, Soviet. Astron. Lett., 10, 177

Bulik, T., Belczynski, K., \& Kalogera, V. 2003, in Gravitational Wave Detection, ed. M. Cruise, \& P. Saulson, Proc. SPIE, 4856, 146

Chow, E., \& Monaghan, J. J. 1997, J. Comput. Phys., 134, 296

Damour, T., Iyer, B. R., \& Sathyaprakash, B. S. 2001, Phys. Rev. D, 63, 044023 Damour, T., Iyer, B. R., \& Sathyaprakash, B. S. 2002, Phys. Rev. D, 66, 027502 Dolag, K., Vazza, F., Brunetti, G., \& Tormen, G. 2005, MNRAS, 364, 753

Eichler, D., Livio, M., Piran, T., \& Schramm, D. N. 1989, Nature, 340, 126 Faber, J., \& Rasio, F. A. 2000, Phys. Rev. D, 62, 064012

Faber, J., \& Rasio, F. A. 2002, Phys. Rev. D, 65, 084042

Faber, J., Rasio, F. A., \& Manor, J. B. 2001, Phys. Rev. D, 63, 044012

Faber, J., Grandclément, P., \& Rasio, F. A. 2004, Phys. Rev. D, 69, 124036

Faye, G., \& Schäfer, G. 2003, Phys. Rev. D, 68, 084001

Fox, D. B., et al. 2005, Nature, 437, 845 
Gehrels, N., et al. 2005, Nature, 437, 851

Gourgoulhon, E., Grandclément, P., Taniguchi, K., Marck, J.-A., \& Bonazzola, S. 2001, Phys. Rev. D, 63, 064029

Grandclément, P., Gourgoulhon, E., \& Bonazzola, S. 2002, Phys. Rev. D, 65, 044021

Houser, J. L., \& Centrella, J. M. 1996, Phys. Rev. D, 54, 7278

Isenberg, J., \& Nester, J. 1980, in General Relativity and Gravitation, ed. A. Held (New York: Plenum Press), 1, 23

Kochanek, C. S. 1992, ApJ, 398, 234

Lattimer, J. M., \& Swesty, F. D. 1991, Nucl. Phys. A, 535, 331

Lattimer, J. M., Pethik, C. J., Ravenhall, D. G., \& Lamb, D. Q. 1985, Nucl. Phys. A, 432, 646

Miller, M. 2004, Phys. Rev. D, 69, 124013

Monaghan, J. J. 1992, ARA\&A, 30, 543

Morris, J. P., \& Monaghan, J. J. 1997, J. Comput. Phys., 136, 41

Morrison, I. A., Baumgarte, T. W., \& Shapiro, S. L. 2004, ApJ, 610, 941

Narayan, R., Paczyński, B., \& Piran, T. 1992, ApJ, 395, L83

Nice, D. J., Splaver, E. M., Stairs, I. H., et al. 2005, ApJ, 634, 1242

Oechslin, R., \& Janka, T. 2006, MNRAS, 368, 1489

Oechslin, R., \& Janka, T. 2007, in preparation

Oechslin, R., Rosswog, S., \& Thielemann, F.-K. 2002, Phys. Rev. D, 65, 103005 Oechslin, R., Uryū, K., Poghosyan, G., \& Thielemann, F.-K. 2004, MNRAS, 349, 1469

Oohara, K., \& Nakamura, T. 1989, Prog. Theor. Phys., 82, 535
Oohara, K., \& Nakamura, T. 1992, Prog. Theor. Phys., 88, 307

Paczyński, B. 1986, ApJ, 308, L43

Paczyński, B. 1991, Acta Astron., 41, 257

Rosswog, S., \& Davies, M. B. 2002, MNRAS, 334, 481

Rosswog, S., Davies, M. B., Thielemann, F. K., \& Piran, T. 2000, A\&A, 360, 171

Rosswog, S., \& Liebendörfer, M. 2003, MNRAS, 342, 673

Rosswog, S., Liebendörfer, M., Thielemann, F. K., et al. 1999, A\&A, 341, 499

Ruffert, M., \& Janka, H.-T. 2001, A\&A, 380, 544

Ruffert, M., Janka, H.-T., \& Schäfer, G. 1996, A\&A, 311, 532

Shen, H., Toki, H., Oyamatsu, K., \& Sumiyoshi, K. 1998a, Nucl. Phys. A, 637, 435

Shen, H., Toki, H., Oyamatsu, K., \& Sumiyoshi, K. 1998b, Prog. Theor. Phys., 100,1013

Shibata, M. 1998, Phys. Rev. D, 58, 024012

Shibata, M., \& Taniguchi, K. 2006, Phys. Rev. D, 73, 064027

Shibata, M., \& Uryū, K. 2002, Prog. Theor. Phys., 107, 265

Shibata, M., Baumgarte, T., \& Uryū, K. 1998, Phys. Rev. D, 58, 023002

Shibata, M., Karino, S., \& Eriguchi, Y. 2003, MNRAS, 343, 619

Shibata, M., Taniguchi, K., \& Uryū, K. 2005, Phys. Rev. D, 71, 084021

Siegler, S., \& Riffert, H. 2000, ApJ, 531, 1053

Stairs, I. H. 2004, Science, 304, 547

Uryū, K., \& Eriguchi, Y. 2000, Phys. Rev. D, 61, 124023

Wilson, J. R., Matthews, G. J., \& Marronetti, P. 1996, Phys. Rev. D, 54, 1317 
R. Oechslin et al.: Relativistic NSM simulations with non-zero temperature EoSs. I., Online Material $p 1$

\section{Online Material}




\section{Appendix A: Hydrodynamics and field equations}

In the following, we briefly summarize the physical and numerical framework we are using. We work with a $3+1$ decomposition of spacetime, using the following form of the metric $\mathrm{d} s^{2}=g_{\mu \nu} \mathrm{d} x^{\mu} \mathrm{d} x^{\nu}$

$\mathrm{d} s^{2}=\left(-\alpha^{2}+\beta_{i} \beta^{i}\right) \mathrm{d} t^{2}+2 \beta_{i} \mathrm{~d} x^{i} \mathrm{~d} t+\gamma_{i j} \mathrm{~d} x^{i} \mathrm{~d} x^{j}$

where $\alpha$ is the lapse function, $\beta^{i}$ is the shift vector, and $\gamma_{i j}$ is the spatial metric.

Imposing the conformally flat condition $\gamma_{i j}=\psi^{4} \delta_{i j}$, where $\psi$ is the conformal factor, and choosing maximal slicing $\partial_{t} K=$ $K=0$, the Einstein equations can be written in the following set of five elliptic equations for the metric elements (see e.g. Wilson et al. 1996; Shibata et al. 1998):

$$
\begin{aligned}
\Delta(\alpha \psi)=2 \pi \alpha \psi^{5}\left(\rho_{E}+2 S\right)+\frac{7}{8} \alpha \psi^{5} K_{i j} K^{i j}, \\
\Delta \psi=-2 \pi \psi^{5} \rho_{E}-\frac{1}{8} \psi^{5} K_{i j} K^{i j} \equiv 4 \pi \S_{\psi}, \\
\Delta \beta^{i}=-\frac{1}{3} \partial^{i} \partial_{j} \beta^{j} \\
+\partial_{j} \ln \left(\frac{\alpha}{\psi^{6}}\right)\left(\partial^{j} \beta^{i}+\partial^{i} \beta^{j}-\frac{2}{3} \delta^{i j} \partial_{l} \beta^{l}\right) \\
+16 \pi \alpha \psi^{4} j^{i} .
\end{aligned}
$$

Here, $K_{i j}$ refers to the extrinsic curvature of the $t=$ const-3-slice and $K=K_{i}^{i}$ denotes its trace. In the conformally-flat-maximalslicing approximation, $K_{i j}$ can be calculated directly from the metric elements

$2 \alpha \psi^{-4} K_{i j}=\delta_{i l} \partial_{j} \beta^{l}+\delta_{j l} \partial_{i} \beta^{l}-\frac{2}{3} \delta_{i j} \partial_{k} \beta^{k}$

Matter contributions enter the equations via the following source terms

$\rho_{E}=n^{\mu} n^{\nu} T_{\mu \nu}$,

$j^{i}=\gamma_{\mu}^{i} n_{\nu} T^{\mu \nu}$,

$S_{i j}=\gamma_{i \mu} \gamma_{j v} T^{\mu v}$,

which refer to the matter energy density, the matter momentum density and the spatial projection of the energy momentum tensor. Here, $n^{\mu}$ denotes the unit normal vector to the $t=$ const-3-slice.

Assuming a perfect fluid with an energy momentum tensor

$T_{\mu \nu}=\rho h u_{\mu} u_{v}+p g_{\mu \nu}$,

the first two matter contribution terms take the following form

$\rho_{E}=\rho h\left(\alpha u^{0}\right)^{2}-p$,

$j^{i}=\rho h \alpha u^{0} u^{\mu} \gamma_{\mu}^{i}$.

Here, $\rho$ is the baryonic rest mass density, $h=1+p / \rho+\epsilon$ the relativistic enthalpy, $p$ the fluid pressure, $\epsilon$ the specific internal energy and $u^{\mu}$ the four velocity of the fluid. The Lorentz factor $W=\left(1+\gamma^{i j} u_{i} u_{j}\right)^{1 / 2}$ can be calculated using the normalisation condition $u_{\mu} u^{\mu}=-1$ :

$\alpha u^{0}=\left(1+\gamma^{i j} u_{i} u_{j}\right)^{1 / 2}$.
The relativistic hydrodynamics equations are implemented in the following form

$$
\begin{aligned}
\frac{\mathrm{d}}{\mathrm{d} t} \rho^{*}= & -\rho^{*} \partial_{i} v^{i}, \\
\frac{\mathrm{d}}{\mathrm{d} t} \hat{u}_{i}= & -\frac{1}{\rho^{*}} \alpha \psi^{6} \partial_{i} p-\alpha \hat{u}^{0} \partial_{i} \alpha+\hat{u}_{j} \partial_{i} \beta^{j}+\frac{2 \hat{u}_{k} \hat{u}_{k}}{\psi^{5} \hat{u}^{0}} \partial_{i} \psi, \\
\frac{\mathrm{d}}{\mathrm{d} t} \tau= & -\frac{p}{\rho} \partial_{i}\left(v^{i}+\beta^{i}\right) \\
& -\frac{1}{\rho^{*}}(1-W h) \partial_{i} p\left(v^{i}+\beta^{i}\right)+S,
\end{aligned}
$$

where

$$
\begin{aligned}
\frac{\mathrm{d}}{\mathrm{d} t}=\partial_{t}+v^{i} \partial_{i} & \\
\hat{u}_{i}= & h u_{i} \\
\rho^{*}= & \rho \alpha u^{0} \psi^{6} \\
v^{i}= & -\beta^{i}+\frac{\delta^{i j} u_{j}}{\psi^{4} u^{0}} \\
\tau= & h W-\frac{p}{\rho W}-1-\frac{1}{2} \gamma^{i j} \hat{u}_{i} \hat{u}_{j}, \\
S= & \frac{\hat{u}_{i} \hat{u}_{j}}{h u^{0}} K^{i j}(1-W h)-\partial_{i} \alpha(1-W h) \psi^{-4} \hat{u}_{i} \\
& -6 \frac{p}{\rho^{*}}\left(v^{i}+\beta^{i}\right) \psi^{5} \partial_{i} \psi
\end{aligned}
$$

While the continuity and momentum equations, Eqs. (A.13) and (A.14), respectively, are commonly used in relativistic hydrodynamics in this form, the energy Eq. (A.15) is constructed out of the more commonly used variant

$$
\begin{aligned}
\frac{\mathrm{d}}{\mathrm{d} t} \tau_{0}= & -\frac{1}{\rho^{*}} \partial_{i}\left(\psi^{6} p\left(v^{i}+\beta^{i}\right)\right) \\
& +\frac{\hat{u}_{i} \hat{u}_{j}}{h u^{0}} K^{i j}-\frac{\hat{u}_{i}}{\psi^{4}} \partial_{i} \alpha
\end{aligned}
$$

together with the momentum Eq. (A.14) and the Einstein equation to describe the time derivative of $\psi$ contained in the spatial metric in expression (A.20). Here, $\tau_{0}=h W-\frac{p}{\rho W}-1$ is the total relativistic energy. In our simulations, Eq. (A.15) yields more accurate results than Eq. (A.22) in regions where the kinetic energy dominates over the internal energy part, e.g. at the surface of the moving NSs or in cold regions of the torus. This is because $\tau$ is close to the internal energy $\epsilon$, while $\tau_{0}$ contains contributions both from the internal and the kinetic energies.

With the above set of hydrodynamics equations, we evolve the quantities $\rho^{*}, \hat{u}_{i}$ and $\tau$ forward in time using a 4th order Runge-Kutta scheme. At each timestep, we have to recover the primitive quantities $\rho, v^{i}$ and $\epsilon$ from the evolved ones $\rho^{*}, u_{i}$ and $\tau$ using Eqs. (A.18)-(A.20). These relations cannot be solved for the primitives in an analytical closed form and have to be solved iteratively.

Since we do not implement any physics changing the electron fraction $Y_{\mathrm{e}}$ in our fluid, the evolution of $Y_{\mathrm{e}}$ is given by

$\frac{\mathrm{d}}{\mathrm{d} t} Y_{\mathrm{e}}=0$

i.e. $Y_{\mathrm{e}}$ is simply advected with the fluid elements.

To integrate Eqs. (A.13)-(A.15) and (A.23), we use the Smoothed particle hydrodynamics (SPH) method. Their implementation in SPH is fairly straightforward as their form is similar to the Newtonian analogue, (see e.g. Siegler \& Riffert 2000; Oechslin et al. 2004). 
The Poisson-type metric Eqs. (A.2)-(A.4) are solved on an overlaid grid with a full multigrid solver. Since the unknowns also appear on the RHS in the source terms, we have to iterate until convergence is reached. Typically, if we start with the values from the previous timestep as an initial guess, three or four iterations are enough. If we start from a flat metric, i.e. $\alpha=\psi=1, \beta^{i}=0$ up to 10 iterations are necessary.

Boundary conditions and the extension beyond the grid are obtained by a multipole expansion to quadrupole order of the formal solution

$\Delta \psi=-4 \pi S_{\psi} \Rightarrow \psi(\boldsymbol{r})=\int S_{\psi}\left(\boldsymbol{r}^{\prime}\right) /\left|\boldsymbol{r}-\boldsymbol{r}^{\prime}\right| \mathrm{d}^{3} \boldsymbol{r}^{\prime}$.

The mapping of the necessary hydrodynamical quantities from the particles to the grid and the metric quantities back to the particles is carried out with a third order interpolation.

\section{A.1. Artificial viscosity scheme}

In the hydrodynamics solver, we have implemented a new artificial viscosity (AV) scheme proposed by Chow \& Monaghan (1997), which is motivated by considering each particleneighbour pair as left and right states of a one-dimensional Riemann problem. The quantities entering the resulting viscous interaction between the two partners $a$ and $b$ are then the differences of the considered physical quantities, projected along the line connecting the two particles $\mathrm{a}$ and $\mathrm{b}$ and a signal velocity indicating the speed of a signal sent from $a$ to $b$, as seen in the computing frame.

Following Chow \& Monaghan (1997), we introduce an additional viscous pressure term $\Pi_{a b}$ in the $S P H$ momentum equation

$\frac{\mathrm{d} \hat{u}_{i, \mathrm{a}}}{\mathrm{d} t}=-\alpha_{\mathrm{a}} \psi_{\mathrm{a}}^{6} \sum_{\mathrm{b}} m_{\mathrm{b}}\left(\frac{p_{\mathrm{a}}}{\rho_{\mathrm{a}}^{* 2}}+\frac{p_{\mathrm{b}}}{\rho_{\mathrm{b}}^{* 2}}+\Pi_{\mathrm{ab}}\right) \partial_{i} W_{\mathrm{ab}}+R_{1}$,

where

$\Pi_{\mathrm{ab}}=-\frac{K v_{\mathrm{sig}}}{\rho_{\mathrm{ab}}^{*}}\left(\hat{u}_{i, \mathrm{a}}^{*}-\hat{u}_{i, \mathrm{~b}}^{*}\right) e^{i}$,

when particles $\mathrm{a}$ and $\mathrm{b}$ are approaching. Otherwise, we set $\Pi_{\mathrm{ab}}=0$. Here, $K$ is a free parameter to adjust the AV strength, $v_{\text {sig }}$ stands for the signal velocity as discussed below, $\boldsymbol{e}=\boldsymbol{r}_{\mathrm{a}}-$ $\boldsymbol{r}_{\mathrm{b}} /\left|\boldsymbol{r}_{\mathrm{a}}-\boldsymbol{r}_{\mathrm{b}}\right|$ is the unit vector from particle $\mathrm{b}$ to particle $\mathrm{a}$ and $\rho_{\mathrm{ab}}^{*}=\left(\rho_{\mathrm{a}}^{*}+\rho_{\mathrm{b}}^{*}\right) / 2$ is the averaged coordinate conserved density. $W_{\mathrm{ab}}$ is the SPH interpolating kernel and the sum in the momentum equations runs over all neighbouring particles. The remaining term $R_{1}$ stands for the contribution arising due to the gravitational interaction. Note that we have slightly adapted our SPH equations to general relativistic hydrodynamics.

To guarantee that the viscous dissipation is positive definite, we replace the usual $\hat{u}_{i}$ by

$\hat{u}_{i}^{*}=h\left(v^{i}+\beta^{i}\right) \psi^{4} u^{0 *}$,

where

$u^{0 *}=W^{*} / \alpha=\left(1+\psi^{-4}\left(u_{i} e^{i}\right)^{2}\right)^{1 / 2} / \alpha$

is calculated with the Lorentz factor $W^{*}$ that involves the specific momentum projected onto the line joining the two particles a and $\mathrm{b}$. To determine the viscous contribution to the change in $\tau$, we first consider the SPH form of the total energy Eq. (A.22):

$$
\begin{aligned}
\frac{\mathrm{d} \tau_{0}}{\mathrm{~d} t}= & -\sum_{\mathrm{b}} m_{\mathrm{b}}\left(\frac{\psi_{\mathrm{a}}^{6} p_{\mathrm{a}}\left(v^{i}+\beta^{i}\right)_{\mathrm{a}}}{\rho_{\mathrm{a}}^{* 2}}+\frac{\psi_{\mathrm{b}}^{6} p_{\mathrm{b}}\left(v^{i}+\beta^{i}\right)_{\mathrm{b}}}{\rho_{\mathrm{b}}^{* 2}}+\Omega_{\mathrm{ab}}^{i}\right) \\
& \times \partial_{i} W_{\mathrm{ab}}+R_{2},
\end{aligned}
$$

where

$\Omega_{\mathrm{ab}}^{i}=-\frac{K v_{\mathrm{sig}}\left(\tau_{0, \mathrm{a}}^{*}-\tau_{0, \mathrm{~b}}^{*}\right) e^{i}}{\rho_{\mathrm{ab}}^{*}}$

if particles a and b are approaching, and $\Omega_{\mathrm{ab}}^{i}=0$ otherwise. Here, $R_{2}$ stands for all additional terms due to gravity, and $\tau_{0}^{*}$ is identical to $\tau_{0}$ with $W$ being replaced by $W^{*}$.

Using

$\frac{\mathrm{d} \tau}{\mathrm{d} t}=\frac{\mathrm{d} \tau_{0}}{\mathrm{~d} t}-\frac{\hat{u}_{i}}{\psi^{4}} \frac{\mathrm{d} \hat{u}_{i}}{\mathrm{~d} t}+2 \psi^{-5} \frac{\mathrm{d} \psi}{\mathrm{d} t}$,

we finally end up, using also $\tau_{0}^{*}=\tau^{*}+\left(\hat{u}_{i} \mathrm{e}^{i}\right)^{2} /\left(2 \psi^{4}\right)$, with the viscous contribution to the change in $\tau$ :

$$
\begin{aligned}
\frac{\mathrm{d} \tau_{\mathrm{a}}}{\mathrm{d} t}= & -\sum_{\mathrm{b}} m_{\mathrm{b}} \frac{K v_{\mathrm{sig}}}{\rho_{\mathrm{ab}}^{*}}\left(\left(\tau_{\mathrm{a}}^{*}-\tau_{\mathrm{b}}^{*}\right)+\frac{\left(\hat{u}_{i, \mathrm{a}} e^{i}\right)^{2}}{2 \psi_{\mathrm{a}}^{4}}-\frac{\left(\hat{u}_{i, \mathrm{~b}} e^{i}\right)^{2}}{2 \psi_{\mathrm{b}}^{4}}\right. \\
& \left.-\frac{\hat{u}_{i, \mathrm{a}} e^{i}\left(\hat{u}_{j, \mathrm{a}}-\hat{u}_{j, \mathrm{~b}}\right) e^{j}}{\psi_{\mathrm{a}}^{4}}\right) e^{k} \partial_{k} W_{\mathrm{ab}}+R_{3},
\end{aligned}
$$

where $R_{3}$ denotes the additional terms from hydrodynamical and gravitational interactions. Since the term $\tau_{\mathrm{a}}^{*}-\tau_{\mathrm{b}}^{*}$ leads to a nonzero viscous contribution even in the case $\boldsymbol{v}_{\mathrm{a}}=\boldsymbol{v}_{\mathrm{b}}$, we omit it in the final AV implementation. Since this term is antisymmetric in $a$ and $b$, this does not lead to a violation of energy conservation.

Finally, the signal velocity $v_{\text {sig }}$ is approximated by

$v_{\mathrm{sig}}=c_{\mathrm{a}}^{\prime}+c_{\mathrm{b}}^{\prime}+\left|v_{\mathrm{ab}}^{*}\right|$,

where $v_{\mathrm{ab}}^{*}=\left(v_{\mathrm{a}}^{i}-v_{\mathrm{b}}^{i}\right) e^{i}$ is the relative speed projected onto the line joining the two particles and

$c_{\mathrm{a}}^{\prime}=\frac{c_{\mathrm{a}}+\left|v_{\mathrm{ab}}^{*}\right|}{1+c_{\mathrm{a}}\left|v_{\mathrm{ab}}^{*}\right|}$

approximates the sound speed as seen in the local frame.

In addition, we implement a time-dependent viscosity parameter $K$ which is increased in the presence of shocks, i.e. in regions where $\partial_{i} v^{i}$ is strongly negative and which decreases exponentially in regions with $\partial_{i} v^{i} \gtrsim 0$ to a constant minimal value (Morris \& Monaghan 1997).

Comparisons of this viscosity scheme with the one used in the old code version (Oechslin et al. 2004) show less particle noise and a smaller numerical viscosity (see also similar work by Dolag et al. 2005 in a cosmological context).

\section{A.2. Radiation reaction and gravitational wave extraction}

The CFC approximation does not include the effects gravitational radiation by construction. We therefore need a scheme to mimic the GW backreaction onto the matter and a GW extraction scheme. Following Faye \& Schäfer (2003), we implement the backreaction scheme by adding a small, non-conformally flat contribution to the metric. We implement their 3.5PN-accurate formalism, take, however, only into account all 2.5PN-terms and the 3.5PN corrections related to the gravitational potential and its derivatives. The reason is that during the inspiral phase, where the backreaction is most important, PN corrections are dominated by the internal gravitational potential $U \simeq\left(\psi^{4}-1\right) / 2 \sim 0.3$ in the two NSs, whereas, e.g., velocity related corrections are considerably smaller. 
The following equations are solved

$\Delta U_{5}=-4 \pi \sigma, \quad \sigma=T^{00}+T^{i i}$,

$\Delta R=-4 \pi I_{i j}^{[3]} x^{i} \partial_{j} \sigma$,

$\Delta U_{7}=-4 \pi \rho^{*}\left(I_{i j}^{[3]} x^{i} \partial_{j} U_{5}-R\right)$,

$h_{00}=-\frac{4}{5}\left(1-2 U_{5}\right)\left(I_{i j}^{[3]} x^{i} \partial_{j} U_{5}-R\right)-\frac{8}{5} U_{7}$,

$h_{i j}=-\frac{4}{5} I_{i j}^{[3]}$,

with (Blanchet et al. 1990)

$$
\begin{aligned}
I_{i j}= & \int \rho^{*}\left\{x^{i} x^{j}\left(1+\frac{v^{2}}{2}-U+\epsilon\right)+\frac{11}{21} r^{2} v^{i} v^{j}\right. \\
& -\frac{4}{7} x^{i} x^{k} v^{j} v^{k}+\frac{4}{21} v^{2} x^{i} x^{j}+\frac{11}{21} r^{2} x^{i} \partial_{j} U \\
& \left.-\frac{17}{21} x^{i} x^{j} x^{k} \partial_{k} U\right\} .
\end{aligned}
$$

Here, $r^{2}=x^{i} x^{i}, v^{2}=v^{i} v^{i}$, and $U$ is the Newtonian gravitational potential. The first time derivative $I_{i j}^{[1]}$ of the quadrupole is calculated analytically using the above approximations and neglecting the total time derivatives of $U, \partial_{i} U, v^{2}, h$. These derivatives are identically zero in the case of, e.g., a corotating binary. In the case of non-corotating NS spins, a small error is introduced. The second and third time derivatives, $I_{i j}^{[2]}$ and $I_{i j}^{[3]}$, respectively, are calculated on-the-fly numerically with a finite difference approximation.

\section{Appendix B: Definitions}

For further use, we define the following quantities.

Angular momentum:

$J=\int \rho^{*} \hat{u}_{\phi} \mathrm{d}^{3} x=\int \rho^{*}\left(x \hat{u}_{y}-y \hat{u}_{x}\right) \mathrm{d}^{3} x$,

total baryonic mass:

$M_{0}=\int \rho^{*} \mathrm{~d}^{3} x$,

total gravitational mass:

$M=2 \int S_{\psi} \mathrm{d}^{3} x$

and the orbital angular velocity:

$\Omega=\frac{\boldsymbol{r}_{\mathrm{CM}} \times \boldsymbol{v}_{\mathrm{CM}}}{r_{\mathrm{CM}}^{2}}$,

where the index "CM" refers to the center of mass.

\section{Appendix C: The torus criterion}

We calculate the specific angular momentum $u_{\phi}$ of a test particle rotating $\left(=\hat{u}_{\phi}\right.$, since $\left.h=1\right)$ on the innermost stable circular orbit (ISCO) around a $\mathrm{BH}$ as follows.

First, we determine the angular velocity $\Omega=\mathrm{d} \phi / \mathrm{d} t$ and specific angular momentum $u_{\phi}$ characterizing a given stationary circular orbit around a BH with a fixed metric $g_{\mu \nu}$ by solving the corresponding geodesic equation $\ddot{x}^{r}+\Gamma_{\mu \nu}^{r} \dot{x}^{\mu} \dot{x}^{v}=0$. For a circular orbit in the equatorial plane, we have $\dot{r}=\dot{\theta}=0$ and $\theta=\pi / 2$ so that the geodesic equation reduces to

$\Gamma_{t t}^{r}+2 \Gamma_{\phi t}^{r} \Omega+\Gamma_{\phi \phi}^{r} \Omega^{2}=0$.

Here, the $\Gamma_{i j}^{k}$ are the Christoffel symbols associated with the $\mathrm{BH}$ metric. This equation specifies the angular velocity.

The specific angular momentum $u_{\phi}$ is then given by

$u_{\phi}=g_{\phi \mu} u^{\mu}$.

We describe the $\mathrm{BH}$ resulting from the collapse of the merger remnant by the following pseudo-Kerr metric (Grandclément et al. 2002)

$\mathrm{d} s^{2}=-\alpha^{2} \mathrm{~d} t^{2}+\psi^{4}\left(r^{2} \sin ^{2} \theta\left(\mathrm{d} \phi-N^{\phi} \mathrm{d} t^{2}\right)^{2}+\mathrm{d} r^{2}+r^{2} \mathrm{~d} \theta^{2}\right)$,

where

$\alpha^{2}=1-\frac{2 M R}{\Sigma}+\frac{4 a^{2} M^{2} R^{2} \sin ^{2} \theta}{\Sigma^{2}\left(R^{2}+a^{2}\right)+2 a^{2} \Sigma M R \sin ^{2} \theta}$,

$\psi^{4}=1+\frac{2 M}{r}+\frac{3 M^{2}+a^{2} \cos ^{2} \theta}{2 r^{2}}+\frac{\left(M^{2}-a^{2}\right) M}{2 r^{3}}$

$$
+\frac{\left(M^{2}-a^{2}\right)^{2}}{16 r^{4}}
$$

$N^{\phi}=\frac{2 a M R}{\Sigma\left(R^{2}+a^{2}\right)+2 a^{2} M R \sin ^{2} \theta}$,

$R=r+\frac{M^{2}-a^{2}}{4 r}+M$

$\Sigma=R^{2}+a^{2} \cos ^{2} \theta$

Here, $M$ and $a$ are the $\mathrm{BH}$ mass and spin parameter. These quantities are identified with the gravitational mass and the spin parameter of the compact merger remnant. We define this object as the set of all fluid elements which are not found to be in the torus.

Equations (C.1) and (C.2) then simplify to

$\Omega=\frac{-g_{\phi t, r} \pm \sqrt{g_{\phi t, r}^{2}-g_{t t, r} g_{\phi \phi, r}}}{g_{\phi \phi, r}}$,
$u_{\phi}=-\frac{g_{\phi \phi} \Omega+g_{\phi t}}{\sqrt{g_{t t}+g_{\phi t} \Omega+2 g_{\phi \phi} \Omega^{2}}}$,

with

$\begin{aligned} g_{t t, r}= & -2 \alpha^{\prime} \alpha+2 \psi^{3} r N^{\phi}\left(2 r N^{\phi}+\psi^{\prime} \psi N^{\phi}\right. \\ & \left.+\psi r N^{\phi^{\prime}}\right), \\ g_{\phi t, r}= & 2 \psi^{3} r\left(4 r N^{\phi} \psi^{\prime}+2 \psi N^{\phi}+\psi r N^{\phi^{\prime}}\right), \\ g_{\phi \phi, r}= & 2 \psi^{3} r\left(2 r \psi^{\prime}+\psi\right) .\end{aligned}$

Here, the prime stands for the derivative w.r.t. the radial coordinate $r$.

Now, $u_{\phi}$ can be minimized along the radial coordinate $r$ to find the ISCO. This procedure has to be iterated a few times, since the remnant mass and spin parameter themselves depend on the separation between remnant and torus and thus on the ISCO. 